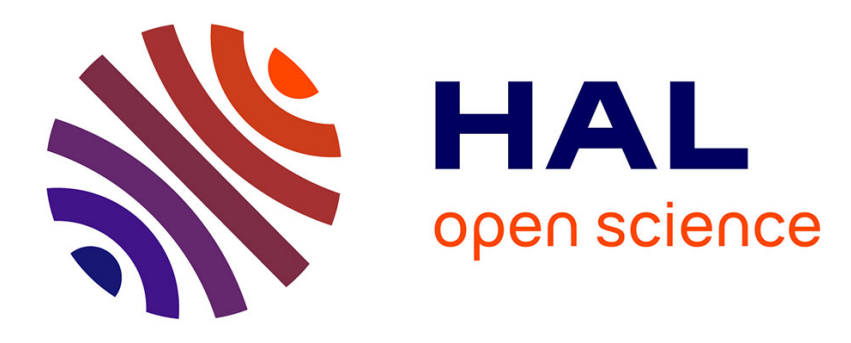

\title{
Repenser l'échec entrepreneurial des petites entreprises émergentes
}

\author{
Ali Smida, Nabil Khelil
}

\section{To cite this version:}

Ali Smida, Nabil Khelil. Repenser l'échec entrepreneurial des petites entreprises émergentes. Revue Internationale PME, 2010, 23 (2), pp.65-106. 10.7202/1005762ar . halshs-02505930

\section{HAL Id: halshs-02505930 \\ https://shs.hal.science/halshs-02505930}

Submitted on 11 Mar 2020

HAL is a multi-disciplinary open access archive for the deposit and dissemination of scientific research documents, whether they are published or not. The documents may come from teaching and research institutions in France or abroad, or from public or private research centers.
L'archive ouverte pluridisciplinaire HAL, est destinée au dépôt et à la diffusion de documents scientifiques de niveau recherche, publiés ou non, émanant des établissements d'enseignement et de recherche français ou étrangers, des laboratoires publics ou privés. 


\title{
« Repenser l'échec entrepreneurial des petites entreprises émergentes : proposition d'une typologie s'appuyant sur une approche intégrative »
}

\author{
Ali Smida et Nabil Khelil \\ Revue internationale P.M.E. : économie et gestion de la petite et moyenne entreprise, vol. $23, \mathrm{n}^{\circ} 2,2010, \mathrm{p}$. \\ 65-106.
}

Pour citer cet article, utiliser l'information suivante :

URI: http://id.erudit.org/iderudit/1005762ar

DOI: 10.7202/1005762ar

Note : les règles d'écriture des références bibliographiques peuvent varier selon les différents domaines du savoir.

Ce document est protégé par la loi sur le droit d'auteur. L'utilisation des services d'Érudit (y compris la reproduction) est assujettie à sa politique d'utilisation que vous pouvez consulter à l'URI http://www.erudit.org/apropos/utilisation.html

Érudit est un consortium interuniversitaire sans but lucratif composé de l'Université de Montréal, l'Université Laval et l'Université du Québec à Montréal. Il a pour mission la promotion et la valorisation de la recherche. Érudit offre des services d'édition numérique de documents scientifiques depuis 1998.

Pour communiquer avec les responsables d'Érudit : erudit@umontreal.ca 


\section{Repenser l'échec entrepreneurial des petites entreprises émergentes Proposition d'une typologie s'appuyant sur une approche intégrative}

Ali SMIDA

Université Paris XIII

Nabil KHELIL

IAE de Caen

MOTS CLÉS

\begin{tabular}{c} 
Échec entrepreneurial - Petite entreprise émergente - \\
$\begin{array}{c}\text { Performance des nouvelles entreprises - Destruction de ressource - } \\
\text { Déception de l'entrepreneur - Discontinuité - Typologie }\end{array}$ \\
\hline
\end{tabular}

\section{LES AUTEURS}

ALI SMIDA détient un doctorat d'État en sciences de gestion, un doctorat en pharmacie, est ingénieur des grandes écoles et diplômé de $3^{e}$ cycle en physique nucléaire. Il est professeur de sciences de gestion à l'Université Paris XIII où il dirige le master «Gestion des entreprises - Management des organisations sanitaires et sociales ". Ses recherches au sein du NIMEC de l'IAE, à l'Université de Caen en France et du CEPN-CNRS de l'Université Paris XIII portent sur la prospective stratégique. En tant que professeur invité, il a animé des séminaires en prospective stratégique dans plusieurs universités en Allemagne, Colombie, France, Pologne, Roumanie, Sénégal et Tunisie. Outre d'être cofondateur du groupe Vision-Prospective de l'IAE de Caen, il est président de l'Association internationale et interdisciplinaire de la Décision (A2ID). II est rédacteur en chef du Zeitschrift für Sozialmanagement/Journal of Social Management et rédacteur en chef associé de Innovar/Social and Management Sciences Journal. Adresse: A2ID, 6, rue Lamartine, 75009 Paris, France. Courriel : <alismida@gmail.com>.

NABIL KHELIL est étudiant au doctorat à l'IAE de Caen Basse-Normandie (NIMEC). En plus d'être membre du centre de recherche à l'École supérieure des sciences économiques et commerciales (ETHICS, Université de Tunis, Tunisie), il est chercheur associé à l'IGS Paris (CR2S-Management). Ses recherches portent sur l'échec entrepreneurial, l'accompagnement psychologique, l'entrepreneuriat chez les jeunes, la cartographie cognitive et la prospective stratégique. Adresse: IAE de Caen Basse-Normandie, NIMEC, 3, rue Claude-Bloch, B.P. 5160, 14075 Caen, France. Courriel : <khelil.nabil@laposte.net>. 


\section{RÉSUMÉ}

Créer une entreprise demeure aujourd'hui une initiative très risquée. Même si les statistiques montrent que la disparition précoce d'une jeune petite entreprise est un destin plus probable que sa survie, la littérature scientifique sur l'entrepreneuriat se concentre sur la réussite entrepreneuriale. Les petites entreprises émergentes qui échouent demeurent un groupe peu étudié. En plus, il n'y a pas de consensus sur la signification de l'échec entrepreneurial qui, d'ailleurs, par sa nature multidimensionnelle et complexe, peut revêtir plusieurs configurations. Dans le but de mieux percevoir l'échec entrepreneurial, le présent article propose une typologie articulée autour des trois théories: la théorie d'écologie des populations des organisations, l'approche fondée sur les ressources et la théorie de la "brèche aspirations-réalisations ". L'écologie des populations des organisations perçoit l'échec en termes de mortalité. Basée sur la primauté des ressources, l'approche du même nom analyse l'échec en termes de défaillance économique. La troisième théorie accorde un rôle important à la motivation entrepreneuriale. Pour la théorie de la "brèche aspirations-réalisations ", l'échec est associé à la déception personnelle du fondateur à la suite de la non-concrétisation de ses aspirations et attentes initiales. À travers la combinaison de ces trois approches, cet article élabore une typologie de huit catégories ou scénarios d'échec. II tente d'aller au-delà de la dichotomie classique qui oppose l' "échec total » et la "réussite absolue ". II introduit les scénarios d' "échec marginal » et d'«échec partiel». Une analyse qualitative exploratoire fondée sur l'étude de 10 cas a servi à illustrer cette typologie.

\section{ABSTRACT}

Starting a new venture remains a very risky initiative. Although statistics show that the disappearance of small businesses is more likely to occur than its survival, specialists have however focused more on entrepreneurial success. There has been very little study on the failure of new ventures. Entrepreneurial failure is a multidimensional and complex phenomenon and, as opposed to seeking a single definition, it is necessary to identify the different types of new ventures that have failed. In effect, this article proposes a typology with the aim of a better perception of entrepreneurial failure. This typology is derived from three theories: the population ecology of organizations, resource-based theory and goal-achievement gap theory. The first approach involving the population ecology of organizations perceives failure in terms of discontinuity. Based on the primacy of resources, the second approach analyzes failure in economic terms. The third approach places emphasis on entrepreneurial motivation. Using the goal-achievement gap theory, failure is associated with the founder's personal disappointment with respect to the non-achievement of her or his specific aspirations and expectations. Combining these three approaches, we have formulated a typology of failure that describes eight categories or scenarios. The purpose of this study is to move beyond the dichotomy between "total failure" and "absolute success" by introducing the notions of "marginal failure" and "partial failure". An exploratory qualitative analysis based on the study of ten cases is used to illustrate this typology. 


\section{RESUMEN}

En la actualidad, crear una empresa continúa siendo una iniciativa de alto riesgo. Aún si las estadísticas muestran que la desaparición precoz de una nueva pequeña empresa es un destino más probable que la supervivencia, los estudios científicos sobre el emprendimiento se concentran en su éxito. El fracaso de las pequeñas empresas emergentes sigue siendo un fenómeno poco estudiado. Adicionalmente, no hay consenso sobre la significación de este concepto en el contexto del emprendimiento, que por su naturaleza multidimensional y compleja, puede tomar varias configuraciones. Con el objeto de mejorar la comprensión del fracaso del emprendimiento, este artículo propone una tipología basada en tres teorías : la ecología de las poblaciones de organizaciones, el enfoque basado en los recursos y la teoría de la "brecha entre aspiraciones y realizaciones". La primera percibe el fracaso en términos de mortalidad, mientras que la segunda lo analiza como la quiebra económica, y la tercera otorga un papel importante a la motivación del emprendedor, asociando el fracaso a la decepción personal del fundador, la cual surge al no haber logrado concretar sus aspiraciones y expectativas iniciales. A través de la combinación de estas tres teorías, en este artículo se elabora una tipología compuesta por ocho categorías o escenarios de fracaso. Tratando de ir más allá de la dicotomía clásica que opone el fracaso total al éxito rotundo, se introducen los escenarios de fracaso marginal y parcial. Un análisis cualitativo exploratorio basado en el estudio de diez casos sirve para ilustrar esta tipología.

\section{ZUSAMMENFASSUNG}

Die Gründung eines Unternehmens ist bis heute mit grossen Risiken verbunden. Obwohl Statistiken zeigen, dass das frühe Verschwinden von Jungunternehmen das wahrscheinlichere Schicksal ist, als dessen Überleben, konzentriert sich die Wissenschaft auf den unternehmerischen Erfolg. Daher stellen kleine Start-ups die scheitern, eine wenig untersuchte Gruppe dar. Darüber hinaus gibt es keinen Konsens über die Bedeutung des unternehmerischen Scheiterns, das in seiner Natur multidimensional und komplex ist und verschiedene Gestalt annehmen kann. Um ein besseres Verständnis der unternehmerischen Misserfolge zu erhalten, schlägt der vorliegende Artikel eine Typologie vor, die auf drei Theorien basiert: der Populationsökologie von Organisationen, dem ressourcenbasierten Ansatz und drittens, der Theorie der Diskrepanz zwischen Bestreben und Realisation. In der Populationsökologie von Organisationen wird Scheitern als Mortalität wahrgenommen. Beim ressourcenbasierten Ansatz ist Scheitern als wirtschaftliches Versagen definiert. Die dritte Theorie schreibt der Motivation eine wichtige Rolle zu. Scheitern also, ist direkt mit der persönlichen Enttäuschung des Unternehmers verbunden, die mit der Nichterreichung der Erwartungen einhergeht. Durch die Kombination dieser drei Ansätze, wurde eine Typologie mit acht Kategorien von Misserfolgsszenarien erstellt. Zudem wird versucht, sich von der klassischen Dichotomie - totales Scheitern und absoluter Erfolg zu entfernen. Zu diesem Zwecke werden neue Begriffe, wie "marginales Versagen" und "teilweises Versagen", eingeführt. Anhand einer explorativen Vorgehensweise bei zehn Fallstudien wurde die neue Typologie illustriert. 


\section{Introduction}

Dans l'environnement turbulent d'aujourd'hui, les entrepreneurs prennent des risques importants à chaque fois qu'ils se lancent dans un projet d'entreprise. Pour beaucoup, la vie de leur entreprise est courte et incertaine. Alors que certains réussissent, d'autres décident d'abandonner après seulement quelques années (Baldwin et al., 2000). En France, comme l'attestent les chiffres de l'Institut national de la statistique et des études économiques (INSEE ${ }^{1}$ ), environ une entreprise sur deux disparaît avant la cinquième année de son existence. Paradoxalement, la plupart des travaux qui s'inscrivent dans le champ de l'entrepreneuriat se concentre sur la réussite entrepreneuriale (Singh, Corner et Pavlovich, 2007) au regard de la rentabilité, de la croissance ou de la survie. Même si les statistiques montrent que la disparition d'une nouvelle entreprise est un destin plus probable que sa survie (Baldwin et al., 2000), les nouvelles entreprises qui échouent demeurent un groupe peu étudié (Bacq, Giacomin et Janssen, 2009; Sammut, 1995, p. 33; Singh, Corner et Pavlovich, 2007).

Les quelques recherches fournies par la littérature donnent des résultats moyens et contradictoires. Cette contradiction s'explique en partie par l'absence d'un consensus sur le concept d'échec entrepreneurial. Pour définir ce concept, l'analyse de ces recherches tend à montrer une forte hétérogénéité. L'appréciation de l'échec ne peut pas être unanime dans la mesure où chaque auteur l'analyse selon l'objectif recherché et l'approche théorique mobilisée. Trouver un critère pour apprécier l'échec et pour le différencier de la réussite demeure une tâche difficile (Moreau, 2007).

Différentes acceptions ont été associées au concept d'échec entrepreneurial (Cope, Cave et Eccles, 2004; McGrath, 1999; Singh, Corner et Pavlovich, 2007). Certains auteurs le jugent à partir de la discontinuité de l'activité entrepreneuriale (Singh, Corner et Pavlovich, 2007). D'autres l'apprécient sur la base de la défaillance économique de la nouvelle entreprise. Malgré la diversité conceptuelle, l'acception la plus répandue tant dans la théorie que dans la pratique est celle qui assimile l'échec à la défaillance et/ou à la faillite (Zacharakis, Meyer et DeCastro, 1999). Dans cette perspective, l'échec entrepreneurial est apprécié à partir des critères de la nonperformance économique privilégiant ainsi une analyse unidimensionnelle du phénomène. Celle-ci ne peut pas aboutir à un consensus puisque l'échec entrepreneurial est un phénomène multidimensionnel et complexe. L'examen de la littérature mène également au constat que rares sont les recherches

1. Selon le tableau de l'économie française (2006), le taux de survie à trois ans est de 63,4 \% ; il est de 50,8 \% à cinq ans pour les entreprises créées en 1998. 
qui proposent une typologie des nouvelles entreprises basées sur les critères d'échec et qui sont centrées sur les petites entreprises émergentes. Ce type d'entreprise semble avoir plus de spécificité par rapport aux entreprises de grande taille qu'elles soient déjà établies ou nouvellement créées (place prépondérante du créateur, décisions centralisées entre les mains de l'entrepreneur-fondateur, les handicaps de petitesse liés à la taille et de nouveauté liés à l'âge, etc.) (Crutzen et Van Caillie, 2008a, 2009; Shelton, 2005; Stinchcombe, 1965).

Dans le but de mieux percevoir l'échec entrepreneurial de ce type d'entreprises, cette étude vise à répondre à la question suivante: «Quelles sont les différentes configurations que peut revêtir l'échec entrepreneurial des petites entreprises émergentes? » Pour répondre à ce questionnement, le présent article propose une typologie articulée autour des trois théories: la théorie d'écologie des populations des organisations, l'approche fondée sur les ressources et la théorie de la «brèche aspirations-réalisations ». La première approche perçoit l'échec en termes de mortalité (Abdesselam, Bonnet et Le Pape, 2004; Chrisman, Bauerschmidt et Hofer, 1999; Dahlqvist, Davidsson et Wiklund, 2000; Fotopoulous et Louri, 2000; Fritsch, Brixy et Falck, 2006; Hannan et Freeman, 1977; Littunen, Storhammar et Nenonen, 1998; Lussier et Pfeifer, 2001 ; Lussier, 1996; Reid, 1999; Stearns et al., 1995; Van Praag, 2003). Dans cette perspective, l'échec est lié à la discontinuité de l'activité entrepreneuriale sous le leadership de l'entrepreneur-fondateur de la nouvelle entreprise. C'est dans ce cadre que s'inscrit la perspective sociologique de la mortalité (Mignon, 2001; Poroli, 1999; Sutton, 1987) qui lie la discontinuité entrepreneuriale à la personne du fondateur. Basée sur la primauté des ressources, la deuxième approche analyse l'échec en termes de défaillance économique (Bacq, Giacomin et Janssen, 2009; Crutzen et Van Caillie, 2008b; Guilhot, 2000; Levinthal, 1991; Malécot, 1991; Mellahi et Wilkinson, 2004; Thornhill et Amit, 2003). La théorie de la «brèche aspirations-réalisations » accorde un rôle important à la motivation entrepreneuriale: elle associe l'échec à la déception personnelle du fondateur à la suite de la non-concrétisation de ses attentes initiales (Cannon et Edmondson, 2001; Cooper et Artz, 1995; Jennings et Beaver, 1995; Murphy et Callaway, 2004). À travers la combinaison de ces trois approches, cet article élabore une typologie de huit catégories ou scénarios d'échec.

Après avoir explicité les sources d'inspiration et les fondements théoriques à l'origine du cadre d'analyse de ce travail (1), cette recherche essaie de mobiliser une approche «poly-théorique» dans une double perspective d'analyse conceptuelle (2) et typologique (3). Enfin, une illustration des apports de cet article est proposée à partir de 10 cas exploratoires (4). 


\section{Cadre d'analyse théorique}

\subsection{Sources d'inspiration}

La littérature qui traite de la prospective, du management stratégique et de l'entrepreneuriat offre des modèles qui peuvent servir de base pour comprendre l'échec des petites entreprises émergentes. On peut citer, à titre indicatif, le modèle SMOCS² (Smida, 1992, 1995, 2006a, 2007), le Factor Coincidence Model (Watkins, 1976), la Configuration stratégique instantanée perçue (Bruyat, 1993, 2001), le modèle des 3E (Paturel, 1997) ainsi que le modèle des 3F (Paturel, 2007; Levy-Tadjine et Paturel, 2006).

S'inspirant de la théorie des ensembles (diagramme de Venn) et s'appuyant sur les concepts de la prospective, Smida (1992,p.59-62) a initialement conçu le modèle SMOCS pour délimiter et étudier les différentes combinaisons d'avenirs. En combinant les trois catégories d'avenirs (les contraints, les possibles et les souhaitables), ce modèle est utilisé pour choisir les stratégies futures destinées à mieux satisfaire les attentes de l'entreprise, à mieux utiliser ses moyens d'action et à faire face à ses contraintes (Smida, 2007). C'est dans ce cadre que s'inscrivent les contributions de ce modèle pour la théorie de la décision. En effet, le modèle SMOCS est utilisé dans plusieurs optiques: pour comprendre les contraintes de la décision (Smida, 2003); pour conceptualiser et modéliser les moments de prise de décision stratégique (Smida, 2006a); pour expliquer l'irrationalité d'une décision stratégique d'une façon générale (Smida, 2006b) et l'irrationalité de la décision entrepreneuriale de création d'entreprises d'une façon particulière (Khelil, 2006).

Par ailleurs, si nous cherchons à analyser l'échec entrepreneurial, nous pouvons utiliser le «Factor Coincidence Model» de Watkins (1976). Ce modèle tente d'expliquer la décision de l'entrepreneur potentiel de créer sa propre entreprise par la coïncidence de trois facteurs: sa motivation entrepreneuriale, sa perception d'une opportunité d'affaires et son savoir-faire. En prenant appui sur ce modèle, Bruyat $(1993,2001)$ s'est intéressé aux éléments stratégiques et psychologiques de la démarche de création d'entreprise. Il définit ainsi la configuration stratégique instantanée perçue (CSIP) par la mise en harmonie et en cohérence des aspirations de l'entrepreneur avec la perception de ses compétences et de ses ressources et avec la perception des opportunités ou des possibilités qu'offre l'environnement.

Pour simplifier l'analyse du phénomène entrepreneurial qui se fonde sur la CSIP, Paturel (1997) a proposé le modèle des 3E. Il explique ainsi l'émergence d'une entreprise ou d'un projet viable par la convergence entre

2. SMOCS: stratégies de moyens, objectifs, contraintes scénarisés. 
les aspirations du créateur (E1), les compétences et ressources intégrées à l'entreprise (E2) et les possibilités de l'environnement (E3). Par ailleurs, le modèle des «3F» (Paturel, 2007; Levy-Tadjine et Paturel, 2006) apporte un éclairage intéressant pour notre analyse conceptuelle. Conçu pour expliquer la performance des PME, il distingue trois dimensions: l'efficacité (F1), qui consiste à approcher les objectifs réalisés des objectifs initialement fixés; l'efficience (F2), qui s'intéresse à la manière dont les ressources et compétences sont utilisées pour réaliser les objectifs de l'entreprise; l'effectivité (F3), qui se concentre sur le degré de satisfaction des parties prenantes de l'entreprise (Paturel, 2007).

En nous appuyant sur l'ensemble de ces travaux, nous relevons les trois dimensions (environnement, ressources de la petite nouvelle entreprise et les motivations de son fondateur) qui constituent, à notre avis, un tout indissociable pour comprendre l'entrepreneuriat. Notre «conceptualisation » s'articule alors autour des éléments suivants: le contexte, les ressources et la motivation. Mais quels sont les fondements théoriques associés à ces trois dimensions?

\subsection{Fondements théoriques}

Pour expliquer l'échec entrepreneurial des petites entreprises émergentes, nous avons distingué trois dimensions clés qui recouvrent, chacune, un ensemble d'approches théoriques. Le premier ensemble d'approches est celui qui accorde un rôle prépondérant au contexte entrepreneurial. Le deuxième ensemble est centré sur la primauté des ressources. Le troisième ensemble privilégie la motivation entrepreneuriale.

\subsubsection{Approches centrées sur la prédominance du contexte entrepreneurial}

Les théories qui s'inscrivent dans le cadre des approches centrées sur la prédominance du contexte adhèrent à la perspective de la dépendance de l'entreprise aux variables environnementales. Ces travaux ont pour caractéristique de mettre l'accent sur l'importance des facteurs contextuels. L'écologie des populations des organisations (EPO) a été l'un des premiers courants de recherche à s'intéresser à l'analyse des déterminants d'échec (Hannan et Freeman, 1977).

Pour expliquer les raisons pour lesquelles certaines nouvelles entreprises échouent et d'autres non, ce courant à forte orientation déterministe (Lelogeais, 2004) accorde un rôle primordial à l'environnement et n'accorde pas de place à l'individu qui est considéré comme un «acteur spectateur» 
(Danjou, 2002). La théorie de l'EPO avance la thèse suivante: le contexte entrepreneurial est une force qui détermine la survivance des entreprises ou, au contraire, leur disparition (Morgan, 2006). Selon les théoriciens de l'EPO, les entreprises sont perçues comme étant totalement et seulement tributaires de leur environnement. Leur viabilité dépend de l'environnement de sélection (Morgan, 2006). Le thème central en est le concept de «sélection environnementale» (Hernandez et Marco, 2002). Dans cette perspective, ce sont les forces contextuelles qui expliquent pourquoi certaines nouvelles entreprises disparaissent et d'autres non. En effet, même si le fondateur possède les compétences essentielles à la réussite, il n'arrive pas à faire survivre son entreprise si le contexte est défavorable. La prédominance du contexte entrepreneurial se manifeste pour le fondateur par la réduction de sa liberté de décider de l'avenir de sa jeune petite entreprise. Quand l'environnement impose un processus de sélection, la disparition de la nouvelle entreprise devient un destin plus probable que sa survie (Baldwin et al., 2000).

Stearns et al. (1995) expliquent l'importance du contexte entrepreneurial par la variété, la dispersion et la disponibilité des ressources qui déterminent les avantages associés à la nature de la localisation géographique de l'entreprise émergente. Du fait de la rareté des ressources, le risque de mortalité infantile est présumé plus élevé dans les agglomérations rurales que dans les agglomérations urbaines (Dahlqvist, Davidsson et Wiklund, 2000; Fotopoulous et Louri, 2000; Fritsch, Brixy et Falck, 2006; Littunen, Storhammar et Nenonen, 1998; Ouedraogo, 1999, p. 178; Stearns et al., 1995).

\subsubsection{Approches centrées sur la primauté des ressources}

Malgré les contributions majeures des approches centrées sur la prédominance du contexte entrepreneurial, en particulier le courant de l'EPO, la plupart des écologistes n'ont pu trouver les raisons pour lesquelles les entreprises qui ont commencé leurs activités au même moment et/ou dans des environnements similaires atteignent des niveaux de performance différents (Cooper, Gimeno-Cacson et Woo, 1994). Pour expliquer la performance des nouvelles entreprises, l'approche fondée sur les ressources met en avant l'importance des moyens internes par rapport aux contraintes externes. Cette approche se fonde sur l'hypothèse selon laquelle la performance dépend largement des ressources dont dispose et que contrôle l'entreprise et qui possèdent certaines caractéristiques particulières (Wernerfelt, 1984): valeur, rareté, inimitabilité et non-substituabilité (Barney,1991). Elle explique également la différence des performances des entreprises par les différences des ressources qu'elles possèdent. 
Pour expliquer la performance de nouvelles entreprises, l'importance des ressources est supportée par plusieurs auteurs. On peut citer, à titre indicatif, les travaux d'Aspelund, Berg-Utby et Skjevdal (2005), Bruton et Rubanik (2002), Cooper, Gascon et Woo (1991) et Thornhill et Amit (2003). Ces derniers avancent que l'échec des entreprises naissantes est un phénomène nettement différent de celui des entreprises déjà établies. En effet, les entreprises naissantes ne se comportent pas aussi bien que les entreprises les plus mûres (Bruton et Rubanik, 2002). Celles-ci échouent parce qu'elles sont incapables de s'adapter à l'érosion des ressources stratégiques causée par le phénomène d'obsolescence. Les petites entreprises émergentes échouent car elles souffrent d'un double handicap de nouveauté et de petitesse (Shelton, 2005 ; Stinchcombe,1965). Ce handicap réduit la capacité de la nouvelle entreprise à survivre lorsqu'elle se trouve en compétition avec des entreprises déjà établies (Aspelund, Berg-Utby et Skjevdal, 2005). Selon l'approche fondée sur les ressources, pour réussir cette sélection, la nouvelle entreprise s'appuie sur la spécificité des ressources qu'elle contrôle, ressources qui, tout en présentant de la valeur pour les clients, sont rares, inimitables et non substituables avec le même prix (Barney, 1991): ce sont des ressources stratégiques. En dehors de ces critères, il y a un risque élevé que la nouvelle entreprise disparaisse (Aspelund, Berg-Utby et Skjevdal, 2005).

En plus de la spécificité des ressources, l'échec entrepreneurial s'explique par la pénurie des ressources vitales qui se manifeste à travers le manque de légitimité, les relations fragiles avec les acteurs externes, le problème d'accès aux canaux de distribution, le manque d'expérience, la sous-capitalisation financière, etc. Parmi ces facteurs, la dotation initiale en capital financier est la plus déterminante de la réussite ou de l'échec (Cooper, Gimeno-Cacson et Woo, 1994; Dahlqvist, Davidsson et Wiklund, 2000; Lussier et Pfeifer, 2001 ; Honjo, 2000; Lasch, Le Roy et Yami, 2005 ; Reid, 1999).

La carence en ressources comme facteur d'échec a aussi été démontrée par Thornhill et Amit (2003). Selon ces auteurs, les chances de réussite des entreprises émergentes dépendent de l'existence d'une dotation initiale en actifs. Le risque d'échec est, par conséquent, plus élevé pour les nouvelles entreprises qui ne possèdent pas suffisamment de ressources pour la mise en œuvre de leur stratégie (Lussier, 1995; Venkataraman et al., 1990). Cooper, Gimeno-Cacson et Woo (1994) avancent l'hypothèse selon laquelle les ressources initiales à la création exercent une influence sur la performance potentielle des nouvelles entreprises. Cette influence subsiste même si les caractéristiques environnementales et organisationnelles changent. À cet effet, les nouvelles entreprises qui bénéficient d'une position avantageuse en termes de ressources occupent une meilleure position pour résister et surmonter les problèmes imprévisibles qui surgissent et qui s'intensifient. 
Les ressources initiales à la création peuvent donc être considérées comme un facteur prédictif du succès des entreprises émergentes. C'est dans ce cadre que s'inscrit le modèle prédictif fondé sur les ressources de Cooper, Gascon et Woo (1991).

\subsubsection{Approches centrées sur l'importance de la motivation entrepreneuriale}

Alors que l'approche fondée sur les ressources explique l'échec des petites entreprises émergentes essentiellement par la spécificité et la pénurie des ressources vitales, d'autres approches mettent l'accent sur le rôle de l'engagement et de la détermination de l'individu. Certaines études ont en effet démontré l'existence d'une relation entre la motivation de l'entrepreneur et la réussite de son entreprise (Wiklund et Shepherd, 2001). Shane, Locke et Collins (2003) admettent que le succès dépend de la volonté et de la motivation des individus. Dans cette perspective, la réussite de l'entrepreneur requiert non seulement un haut niveau de qualification, mais aussi une motivation et une intention forte (Lasch, Le Roy et Yami, 2005). La motivation entrepreneuriale a été considérée comme l'un des éléments les plus importants influençant non seulement le démarrage de la nouvelle entreprise, mais également ses caractéristiques, sa survie, sa performance (Shane, Locke et Collins, 2003) et le niveau de satisfaction de son fondateur (Cooper et Artz, 1995). C'est dans ce cadre que s'inscrit la «goal achievement gap theory» que nous pouvons traduire par la théorie de la «brèche aspirations-réalisations».

La documentation sur le sujet autorise à penser que les entrepreneurs qui ont choisi d'une façon délibérée l'entrepreneuriat parce qu'ils veulent être entrepreneurs (Gray, Foster et Howard, 2006) éprouvent une forte intention. Leurs chances de réussite sont présumées plus élevées (Amit et Muller, 1994). À contrario, ceux qui ont été contraints de créer leur propre entreprise parce qu'ils manquent de travail ou pour gagner de quoi vivre (Gray, Foster et Howard, 2006) expriment une faible volonté. Le risque d'échec est estimé plus élevé pour ce groupe d'entrepreneurs.

La motivation de l'entrepreneur désigne également son engagement (Shane, Locke et Collins, 2003). Les études menées par Murphy et Callaway (2004) démontrent que la réussite entrepreneuriale est sensiblement liée à l'engagement du fondateur. En effet, les entrepreneurs fortement engagés expriment des liens émotifs forts à leur entreprise. Ils sont généralement moins disposés à vendre ou clôturer leurs entreprises indépendamment de la performance économique réalisée. Ils sont prêts à investir leurs biens financiers et leurs énergies pour soutenir le développement et la survie de leur affaire. Cependant, les entrepreneurs qui sont moins engagés se concentrent 
plus sur les coûts d'opportunité. Ils voient dans la performance financière un facteur critique pour décider de la continuité ou de la discontinuité de leur affaire. Ils sont donc à tout moment prêts à vendre ou à clôturer leurs entreprises pour saisir une autre opportunité plus intéressante (Murphy et Callaway, 2004; Papadaki et Chami, 2002).

Le cadre théorique explicité précédemment permet de classer les causes d'échec des petites entreprises émergentes en trois catégories: contexte, ressources et motivation. Ces catégories permettent de distinguer les entreprises qui réussissent de celles qui échouent. Toutefois, comprendre l'échec entrepreneurial reste une tâche difficile essentiellement parce que ce concept n'est pas clairement défini.

\section{Vers une approche «poly-théorique» de l'échec entrepreneurial}

Malgré de nombreuses contributions majeures, le champ de l'entrepreneuriat demeure traversé par des débats divergents concernant la définition et la problématique de l'échec entrepreneurial. La question est donc de savoir «ce que signifie l'échec entrepreneurial» (Singh, Corner et Pavlovich, 2007). Plusieurs définitions ont été proposées qui dépendent de la perspective juridique, économique, financière, stratégique, managériale... (Guilhot, 2000).

Dans les prolongements des travaux récents sur l'entrepreneuriat et en nous appuyant sur une approche «poly-théorique», nous proposons une vision globale de l'échec entrepreneurial des petites entreprises émergentes, une vision intégrative qui prend en compte les dimensions constitutives du phénomène. Nous nous appuyons sur la théorie de l'écologie des populations des organisations, sur l'approche fondée sur les ressources et sur la théorie de la «brèche aspirations-réalisations» pour essayer de clarifier la notion d'«échec entrepreneurial».

\subsection{Approche de l'échec par la théorie d'écologie des populations des organisations}

Selon la théorie de l'EPO, la réussite d'une entreprise s'exprime en termes de survie. Les entrepreneurs qui réussissent sont ceux qui arrivent à faire survivre leur entreprise sur une période plus ou moins longue. S'inscrivant dans cette optique, et pour opérationnaliser la réussite des nouvelles entreprises, plusieurs auteurs utilisent la survie comme indicateur (Abdesselam, Bonnet et Le Pape, 2004 ; Aspelund, Berg-Utby et Skjevdal, 2005 ; Chrisman, 
Bauerschmidt et Hofer, 1999; Dahlqvist, Davidsson et Wiklund, 2000; Littunen, Storhammar et Nenonen, 1998; Lussier et Pfeifers, 2001; Lussier, 1996; Moreau, 2007; Reid, 1999; Van Praag, 2003).

En effet, pour mesurer l'incidence du contexte local sur la réussite entrepreneuriale, Littunen, Storhammar et Nenonen (1998) avancent que la continuité de l'activité entrepreneuriale est le meilleur indicateur de succès. Il y a certes plusieurs définitions du succès, mais ces auteurs suggèrent de rapprocher la conception de réussite à la survie de la nouvelle entreprise. Parce que les premières années sont critiques pour la stabilisation de l'activité entrepreneuriale (Littunen, Storhammar et Nenonen, 1998), Moreau (2007) avance qu'on ne peut parler de réussite que lorsque la nouvelle entreprise se maintient sur le marché pendant une durée de trois ans au moins. Capiez et Hernandez (1998) soutiennent aussi que la mesure de base de la performance des entreprises en émergence est d'abord la survie. Cette position repose sur le constat que seulement $36,6 \%$ des nouvelles entreprises atteignent le seuil de trois ans (Tableau de l'économie française, 2006).

Par opposition, les entrepreneurs qui échouent sont ceux qui n'arrivent pas à maintenir leur entreprise en vie (Chrisman, Bauerschmidt et Hofer, 1999). Dans cette optique, l'échec est assimilé à une mort organisationnelle. C'est dans ce cadre que s'inscrit l'approche de l'échec par la mortalité.

La réflexion sur la mortalité a véritablement commencé à partir des années 1970 avec les travaux de Hannan et Freeman (1977). Selon la théorie de l'EPO, l'échec est synonyme de «mort organisationnelle», de «sortie organisationnelle», de «faillite» (Mellahi et Wilkinson, 2004). Les travaux qui s'inscrivent dans ce courant de recherche identifient généralement la mort d'une organisation à sa mort juridique formelle (Poroli, 1999). L'échec d'une entreprise est donc associé à sa disparition du registre de commerce. Selon Honjo (2000), la forme la plus usuelle de l'échec est celle de la faillite. Pour Singh, Corner et Pavlovich (2007), la faillite est la mesure la plus objective; elle est appropriée pour opérationnaliser l'échec car elle repose sur une manifestation juridique du phénomène. Selon cette optique, l'échec entrepreneurial est associé à une mort juridique.

Dans certaines circonstances, la mort juridique ne coïncide pas obligatoirement avec la mort de l'entité sociologique qu'est l'organisation. Une organisation peut en effet changer de dénomination sociale pour se réorganiser sous la forme d'une nouvelle entité juridique, avec la totalité ou seulement une partie des membres de l'organisation mourante (Poroli, 1999). Évoquer la notion d'identité conduit donc à s'interroger sur la mort sociologique de la nouvelle entreprise: la notion d'identité n'est-elle pas le fil directeur permettant de déterminer la mortalité d'une organisation? (Mignon, 2001.) 
Les recherches menées par Sutton (1987) permettent de donner un éclairage intéressant pour définir la mortalité sociologique. Après une étude empirique qualitative fondée sur huit études de cas, Sutton (1987) décrit le processus menant à la mort en se focalisant sur la relation entre les organisations mourantes et leurs membres. Il spécifie trois composantes: la lutte pour la survie; la dissolution de l'arène organisationnelle «disbanding » et la réinsertion des composantes de l'organisation dans d'autres systèmes sociaux «reconnecting». Dans cette perspective, il semble qu'on ne peut parler de mortalité que lorsque l'entreprise n'a pas su préserver son identité.

Toutefois, il est difficile d'établir la mort des petites entreprises émergentes en se fondant sur leur identité organisationnelle. Celle-ci apparaît comme un ensemble de caractéristiques qui donne à l'entreprise, au cours de son existence, sa spécificité, sa stabilité et sa cohérence (Mignon, 2001, p. 43). Mais il faut du temps pour qu'une entreprise en émergence «construise» sa propre identité. Par ailleurs, il est difficile de distinguer l'identité d'une jeune petite entreprise de celle de son fondateur. Les entreprises récemment créées sont généralement reconnues à travers le nom de leur fondateur. Ce dernier peut cependant mettre fin à son aventure entrepreneuriale. Selon une perspective sociologique de la mortalité, l'échec est lié à la discontinuité de l'activité entrepreneuriale sous le leadership de l'entrepreneur-fondateur de la nouvelle entreprise.

En nous appuyant sur la théorie de l'EPO, la mesure de base du succès des petites entreprises émergentes est la survie. L'échec est alors associé à la discontinuité entrepreneuriale. Cependant, ce critère ne permet pas de différencier les firmes déjà existantes (Capiez et Hernandez, 1998). Par ailleurs, se limiter à la seule notion de survie n'exclut pas de l'espace des échecs les entreprises créées qui demeurent petites, peu profitables (Moreau, 2007), voire défaillantes. Pour pallier cette limite, les chercheurs ont suggéré de définir le succès ou l'insuccès des nouvelles entreprises à partir des critères économiques de la performance (Capiez et Hernandez, 1998). C'est dans ce cadre que s'inscrit l'approche économique de l'échec par la théorie des ressources.

\subsection{Approche économique de l'échec par la théorie des ressources}

Selon l'approche fondée sur les ressources, le succès des entreprises est analysé en termes de rente économique et d'avantage concurrentiel. Les entreprises qui réussissent sont celles qui arrivent à générer une rente suffisante pour créer un avantage concurrentiel durable (Arrègle, 2006). Par opposition, l'échec est analysé en termes d'érosion des ressources et de désavantage concurrentiel. Malgré l'absence d'une définition précise de l'échec, Mellahi et Wilkinson 
(2004) avancent qu'il y a un large consensus sur le sens: il s'agit de la détérioration de la capacité adaptative de l'organisation à une micro-niche associée à une dépréciation des ressources. Dans cette perspective, la détérioration des ressources de l'entreprise est la manifestation d'un échec économique. Selon cet angle d'approche, la conception d'échec est généralement associée à celle de la défaillance économique. Au sens restreint du terme, une entreprise est défaillante si elle est en état de cessation de paiement. Sur le plan juridique, la cessation de paiement signifie que l'entreprise est dans l'incapacité de faire face avec les actifs disponibles au passif immédiatement exigible (Guilhot, 2000). Malécot (1991) ajoute que la cessation de paiement repose aussi sur le refus des créanciers de contribuer à un nouveau financement, voire simplement de donner un délai supplémentaire.

Crutzen et Van Caillie (2008b, 2009) proposent cependant une conception plus large. Ils associent la défaillance au processus de détérioration des ressources. Contrairement aux recherches qui utilisent les termes de défaillance et de faillite de façon équivalente (Malécot, 1991), cette vision de l'échec nous conduit à distinguer ces deux conceptions. Alors que la faillite est une situation ponctuelle caractérisée par une double crise de solvabilité et de liquidité, la défaillance est un processus de détérioration progressive des ressources qui, à défaut d'actions correctives, peut conduire l'entreprise à une faillite (Crutzen et Van Caillie, 2008b, 2009). La faillite est donc la forme la plus extrême d'échec (Thornhill et Amit, 2003).

Ainsi, selon l'approche fondée sur les ressources, l'échec est apprécié sur la base de la défaillance (ou de la non-performance) économique (Lelogeais, 2004). La destruction et le gaspillage des ressources, la profitabilité négative, le désavantage concurrentiel, les crises de solvabilité et de liquidité sont les manifestations économiques d'un échec organisationnel (Mellahi et Wilkinson, 2004). Sur ce point, Levinthal (1991) précise que les entreprises échouent lorsque de mauvais résultats érodent leur stock de ressources. La détérioration des ressources n'englobe pas seulement les actifs financiers mais aussi la position de l'entreprise sur le marché (Thornhill et Amit, 2003).

Apprécier l'échec des petites entreprises émergentes sur la base de critères économiques est une tâche particulièrement difficile pour plusieurs raisons: les entreprises récemment créées ont un historique limité; les créations à succès n'atteignent souvent une rentabilité suffisante qu'après plusieurs années; le taux de croissance des ventes n'est guère significatif les premières années, puisque ces firmes partent de zéro (Capiez et Hernandez, 1998). La question est encore plus compliquée par la difficulté d'obtention de données objectives sur la performance économique. En effet, le fait de demander des informations sensibles ou difficiles peut réduire le taux de 
réponses, faisant ainsi de la collecte des données une tâche difficile (Murphy et Callaway, 2004). Pour surmonter ces difficultés, plusieurs chercheurs ont suggéré l'utilisation des mesures subjectives telles que la satisfaction du fondateur comme une alternative des mesures économiques. C'est dans ce cadre que s'inscrit l'approche psychologique de l'échec.

\subsection{Approche psychologique de l'échec par la théorie de la «brèche aspirations-réalisations »}

Selon l'approche de la performance par la théorie des parties prenantes, le succès et l'échec sont généralement définis sur la base de la satisfaction des principales attentes des parties prenantes (Jennings et Beaver, 1995). Étant donné la nature même de l'organisation entrepreneuriale, il semble approprié de considérer son fondateur comme la partie prenante centrale (Sammut, 1995, p. 31). Parmi les critères d'appréciation de la réussite des entreprises naissantes, on peut retenir ceux qui sont liés à la satisfaction de l'entrepreneur (Cooper et Artz, 1995; Murphy et Callaway, 2004).

Selon Cooper et Artz (1995), la satisfaction de l'entrepreneur est une mesure pertinente de la performance et elle est déterminante pour décider du sort de son entreprise. Elle est considérée comme un facteur qui joue un rôle important dans la décision de poursuivre ou d'arrêter l'activité entrepreneuriale. Elle peut donc affecter la performance de sa jeune petite entreprise sur le long terme. Murphy et Callaway (2004) ont démontré qu'il y a une équivalence entre la satisfaction individuelle comme mesure subjective de la performance et les mesures relativement plus objectives de cette même performance. La satisfaction comme aspect de la réussite a aussi été démontrée dans l'étude menée par Ray et Trupin (1989) portant sur des créateurs d'entreprises technologiques canadiens, américains, japonais et français. Bien que des différences apparaissent dans la manière dont les entrepreneurs évaluent leur succès, il ressort que plus de la moitié d'entre eux évaluent leur réussite en comparant ce qu'ils sont aujourd'hui à ce qu'ils voulaient être lorsqu'ils avaient créé leur entreprise.

Pour conceptualiser la satisfaction entrepreneuriale, Cooper et Artz (1995) ont utilisé la théorie de la «brèche aspirations-réalisations», reconnue dans la littérature anglo-saxonne sous le vocable de "goal achievement gap theory», comme cadre d'analyse. Initialement mobilisée dans la psychologie industrielle et organisationnelle, cette théorie est utilisée pour étudier les déterminants de la satisfaction au travail. L'une des explications que cette théorie suggère est que la satisfaction individuelle est déterminée par l'«écart» entre ce qu'on pense gagner et ce qu'on gagne réellement. L' «écart» entre les gains attendus et les gains effectifs détermine le degré de satisfaction 
ou d'insatisfaction d'un individu (Michalos, 1986; cité par Cooper et Artz, 1995). Cette théorie suppose donc que la satisfaction de l'entrepreneur est déterminée, en partie, par l'écart entre la situation (financière, sociale...) que lui procure réellement sa jeune petite entreprise et ses motivations ou ses attentes initiales lors de la création.

Selon la théorie de la «brèche aspirations-réalisations», les entrepreneurs qui ont réussi sont donc ceux qui sont satisfaits par la concrétisation de leurs aspirations. À contrario, les entrepreneurs qui ont échoué sont ceux qui sont insatisfaits du fait qu'ils n'arrivent pas, à travers leur projet d'entreprise, à exaucer leurs souhaits. Dans cette perspective, l'échec est jugé à partir de la déception personnelle du fondateur à la suite de la non-concrétisation de ses attentes initiales. C'est dans ce cadre que s'inscrit la dimension psychologique de l'échec centrée sur la motivation intrinsèque de l'entrepreneur. Optant pour la même vision, Cannon et Edmondson (2001) définissent l'échec comme une déviation par rapport aux résultats désirés. Pour un entrepreneur, l'échec peut être effectif lorsqu'il n'arrive pas à satisfaire ses besoins de pouvoir ou d'indépendance ou encore de richesse (Moreau, 2007).

TABLEAU 1

Les trois dimensions d'échec et les fondements théoriques associés

\begin{tabular}{|c|c|c|c|}
\hline $\begin{array}{l}\text { Fondements } \\
\text { théoriques }\end{array}$ & $\begin{array}{l}\text { Théorie d'écologie } \\
\text { des populations des } \\
\text { organisations }\end{array}$ & $\begin{array}{l}\text { Approche fondée } \\
\text { sur les ressources }\end{array}$ & $\begin{array}{l}\text { Théorie de la } \\
\text { «brèche aspirations- } \\
\text { réalisations» }\end{array}$ \\
\hline $\begin{array}{l}\text { Conception } \\
\text { de l'échec }\end{array}$ & $\begin{array}{l}\text { Discontinuité } \\
\text { entrepreneuriale } \\
\text { (l'entreprise } \\
\text { émergente n'arrive } \\
\text { pas à survivre au-delà } \\
\text { d'une période de } 3 \text { ans } \\
\text { sous le leadership } \\
\text { de son fondateur) }\end{array}$ & $\begin{array}{l}\text { Défaillance économique } \\
\text { Destruction de } \\
\text { ressources (inefficience, } \\
\text { non-génération } \\
\text { d'une rente suffisante } \\
\text { pour maintenir un } \\
\text { avantage concurrentiel) }\end{array}$ & $\begin{array}{l}\text { Insatisfaction } \\
\text { de l'entrepreneur } \\
\text { (non-concrétisation } \\
\text { de ses aspirations et } \\
\text { attentes initiales) }\end{array}$ \\
\hline $\begin{array}{l}\text { Déterminant } \\
\text { d'échec }\end{array}$ & $\begin{array}{l}\text { Facteurs } \\
\text { environnementaux } \\
\text { inhérents au contexte } \\
\text { de création }\end{array}$ & $\begin{array}{l}\text { Carences en ressources } \\
\text { Pénurie des moyens } \\
\text { internes }\end{array}$ & $\begin{array}{l}\text { Manque } \\
\text { de motivation } \\
\text { et de détermination } \\
\text { à la réussite }\end{array}$ \\
\hline
\end{tabular}

Une étude de la littérature nous a ainsi permis de distinguer trois dimensions de l'échec (tableau 1). Chaque dimension est caractérisée par ses fondements théoriques, sa conception d'échec et les déterminants de cet échec. La vision selon une dimension particulière privilégie un aspect de l'échec au détriment des deux autres dimensions. Le résultat est qu'aucune des trois dimensions prises séparément n'est tout à fait satisfaisante: elle 
souffre des limites que les autres approches essaient de combler. Nous proposons, quant à nous, une approche intégrative de ces trois dimensions dans le but d'apporter une réponse globale à la problématique de la compréhension de l'échec des petites entreprises émergentes.

\section{Les scénarios ou catégories d'échec}

Le recours respectif à la théorie d'écologie des populations des organisations, à l'approche fondée sur les ressources et à la théorie la «brèche aspirationsréalisations » fait apparaître trois dimensions de l'échec: la discontinuité entrepreneuriale (D), la défaillance économique en termes de destruction de ressources (E) et l'insatisfaction (ou déception personnelle) de l'entrepreneur (I). La combinaison de ces trois dimensions d'échec permet de dégager une typologie de huit catégories ou scénarios d'échec. Ainsi nous avons opposé l'«échec total» à la «réussite entrepreneuriale» (tableau 2). Entre ces deux catégories extrêmes, nous distinguons les catégories d' «échec partiel» et d'«échec marginal» (tableaux 3 et 4 ).

\subsection{Les cas extrêmes d'échec total et de réussite entrepreneuriale}

Avant d'expliciter les différentes catégories ou scénarios, il convient de définir les trois espaces d'échec: $\mathrm{S}_{\mathrm{D} \text { (discontinuité) }}, \mathrm{S}_{\mathrm{E} \text { (destruction de ressources) }}$ et $\mathrm{S}_{\mathrm{I} \text { (insatisfaction de }}$ l'entrepreneur) (figure 1, page suivante). L'espace $S_{D}$ regroupe les entreprises nouvellement créées qui ne parviennent pas à survivre et à franchir le cap des trois premières années sous le contrôle de leur fondateur. L'espace $S_{E}$ regroupe les nouvelles entreprises qui détruisent plus de ressources qu'elles ne produisent de richesses. Enfin, l'espace $\mathrm{S}_{\mathrm{I}}$ regroupe les entreprises dont le fondateur est déçu du fait qu'il n'arrive pas, à travers son projet d'entreprise, à concrétiser ses aspirations et ses attentes initiales.

La catégorie de scénarios d'échec total $\mathbf{S}_{\mathrm{DIE}}$. Cette catégorie (tableau 2) se définit par la conjugaison des trois dimensions d'échec: discontinuité entrepreneuriale $\left(\mathrm{S}_{\mathrm{D}}\right)$, destruction de ressources $\left(\mathrm{S}_{\mathrm{E}}\right)$ et déception du fondateur $\left(\mathrm{S}_{\mathrm{I}}\right)$. Les entreprises qui connaissent l'échec total sont celles qui appartiennent à l'espace «Die». Cette situation s'explique essentiellement par la mort de la petite entreprise émergente qui ne génère pas de rente suffisante pour assurer sa survie et dont l'entrepreneur est insatisfait car elle ne répond pas à ses attentes. Ce type d'échec décrit le cas d'une nouvelle entreprise qui a échoué sur tous les plans. Sur un plan économique, l'entreprise émergente disparaît en totalité non seulement sans aucune création de valeur, mais, pis encore, avec une destruction de ressources. Sur le plan psychologique, l'entrepreneur est très insatisfait. Déçu des résultats économiques comparés 


\section{FIGURE 1}

\section{Vers une approche intégrative de l'échec entrepreneurial}

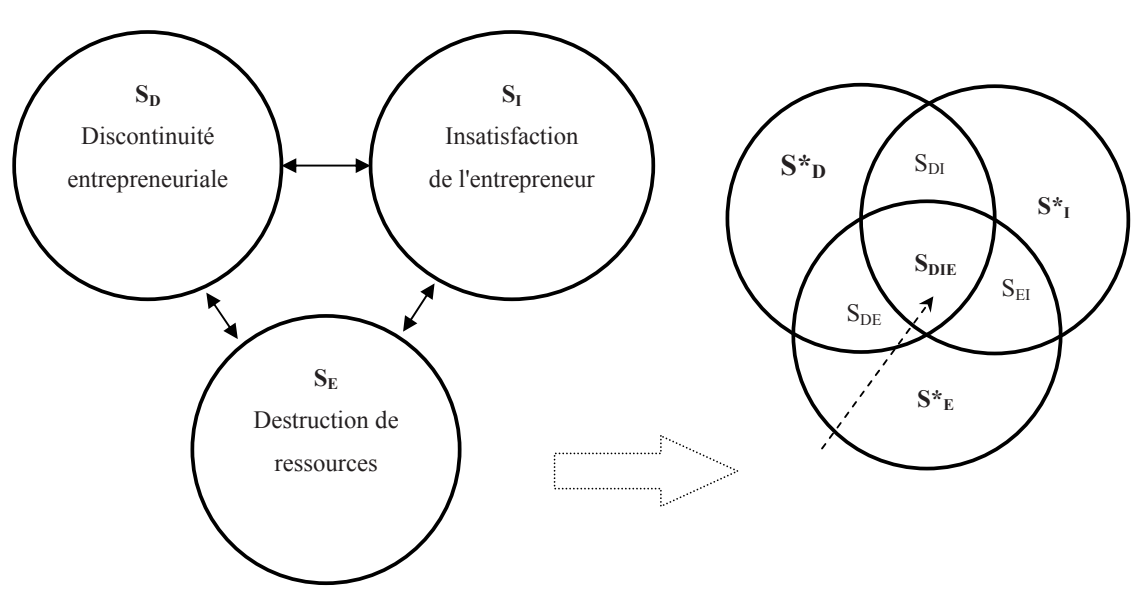

à ceux attendus, il admet qu'il est difficile, à travers son projet d'entreprise, de réaliser ses objectifs personnels. Il décide donc d'abandonner son activité entrepreneuriale. Ce type d'échec peut être qualifié de «sortie négative»: la nouvelle entreprise disparaît en totalité, elle est liquidée en laissant un passif important. Par ailleurs, démoralisé par son échec individuel, le fondateur perd sa légitimité, sa réputation et son identité d'entrepreneur. Dans ce cas d'échec, la mort de l'identité sociologique de la nouvelle entreprise (qui est généralement reconnue à travers le nom de son fondateur) coïncide avec sa mort juridique formelle (disparition du registre de commerce).

La catégorie de scénarios de réussite entrepreneuriale. En dehors des trois espaces d'échec définis précédemment, on retrouve le cas de succès total ou d' «échec zéro» (tableau 2). Cette catégorie se définit par la conjugaison des trois dimensions de succès: continuité entrepreneuriale, création de ressources et satisfaction du fondateur. L'entreprise nouvellement créée est vivante et en bonne santé, génère une rente suffisante, et l'entrepreneur est satisfait car il considère qu'il est en train de réaliser ses attentes initiales. L'entrepreneur parvient à créer une entreprise pérenne qui dégage une rente économique importante pour soutenir le développement de son affaire et qui satisfait à ses exigences personnelles et sociales. 
TABLEAU 2

Les cas extrêmes d'échec total et de réussite entrepreneuriale

\begin{tabular}{ll}
\hline Typologies d'échec & \multicolumn{1}{c}{ Définitions } \\
\hline Échec total $\left\{\mathbf{S}_{\mathbf{D I E}}\right\}$ & Ce cas d'échec s'explique à la fois \\
par la mort de l'entreprise, par la \\
non-génération d'une rente suffi- \\
sante pour assurer la continuité \\
entrepreneuriale et par la non- \\
de ressources \\
concrétisation des aspirations et \\
des attentes de l'entrepreneur. Ce \\
type d'échec décrit la situation au \\
sein de laquelle la nouvelle entre- \\
prise a échoué sur tous les plans. \\
$\begin{array}{l}\text { Il correspond généralement à une } \\
\text { mort par liquidation lors d'une } \\
\text { faillite juridique. }\end{array}$ \\
$\begin{array}{l}\text { L'entrepreneur est parvenu à créer } \\
\text { une entreprise avec un fort poten- } \\
\text { tiel de croissance. Il s'agit d'une } \\
\text { entité qui dégage une rente éco- } \\
\text { entrepreneuriale } \\
\text { «échec zéro» }\end{array}$ \\
$\begin{array}{l}\text { nomique suffisante pour soutenir } \\
\text { le développement de son affaire et } \\
\text { qui satisfait les attentes de l'entre- } \\
\text { preneur. }\end{array}$ \\
$\begin{array}{l}\text { Création } \\
\text { de ressources }\end{array}$
\end{tabular}

Entre l'échec total et la réussite entrepreneuriale, il y a des cas d'échec qui peuvent appartenir à un seul des espaces d'échec précédemment définis (figure 1). Nous parlons alors de catégories ou scénarios d' "échec marginal» (tableau 3). Nous distinguerons les scénarios $S^{*}{ }_{D},{ }^{*}{ }_{I}$ et $S^{*}{ }_{E}$.

\subsection{Les cas d'échec marginal}

La catégorie de scénarios $\mathbf{S}_{\mathbf{D}}^{*}$ ou «sortie positive» . Les entreprises émergentes de cet espace d'échec réussissent économiquement mais disparaissent pour plusieurs raisons autres que financières : l'entrepreneur cède son entreprise à une autre personne, il trouve un emploi suffisamment rémunéré, il part à la retraite... Quels que soient les motifs de cessation d'activité,l'entrepreneur arrive à atteindre ses objectifs personnels. Headd (2003) qualifie ce phénomène d'échec de «sortie positive». L'entrepreneur peut en effet concevoir a priori une «stratégie de sortie» pour faciliter le passage à d'autres situations plus intéressantes. Certes, pour réaliser le succès, l'entrepreneur s'efforce de faire survivre son entreprise, mais, dans certains cas, il choisit la «sortie positive »: alors que son entreprise génère des bénéfices, il envisage l'abandon de son affaire ou sa cession à une autre personne (Headd, 2003). 


\section{La catégorie de scénarios $S_{I}^{*}$ ou «survie avec déception de l'entrepre-} neur ».L'entreprise nouvellement créée arrive à générer une rente suffisante, sa continuité est assurée. Mais, malgré cette réussite de son entreprise,l'entrepreneur n'arrive pas à concrétiser ses aspirations et attentes personnelles. Selon une optique centrée sur la dialogique entrepreneur/entreprise, Bruyat (1993) décrit cette forme d'échec par la situation où la réussite de l'entreprise (réussite sur le plan professionnel) est modérée par des difficultés familiales subies par le fondateur (échec sur le plan personnel). Bien que son entreprise soit prospère, le fondateur n'est pas satisfait de la réalisation de ses aspirations personnelles. Il n'arrive pas à réaliser un équilibre entre son travail et sa vie personnelle et familiale.

\section{TABLEAU 3}

\section{Les cas d'échec marginal}

\begin{tabular}{|c|c|c|}
\hline Typologies d'échec & Définitions & Configurations \\
\hline $\begin{array}{l}\text { Sortie positive }\left\{\mathbf{S}_{\mathbf{D}}^{*}\right\} \\
\text { Discontinuité } \\
\text { Destruction } \\
\text { de ressources } \\
\text { Satisfaction }\end{array}$ & $\begin{array}{l}\text { Cet espace d'échec décrit la situa- } \\
\text { tion dans laquelle l'entrepreneur } \\
\text { conçoit a priori une stratégie de } \\
\text { sortie pour faciliter le passage } \\
\text { à d'autres activités jugées par } \\
\text { lui plus intéressantes. Il décide } \\
\text { d'abandonner ou de céder son } \\
\text { affaire alors qu'elle génère des } \\
\text { bénéfices. }\end{array}$ & \\
\hline $\begin{array}{l}\text { Survie avec déception } \\
\text { de l'entrepreneur }\left\{\mathbf{S}_{1}^{*}\right\} \\
\text { Continuité } \\
\text { Création } \\
\text { de ressources } \\
\text { Insatisfaction }\end{array}$ & $\begin{array}{l}\text { Malgré la réussite économique } \\
\text { de la nouvelle entreprise, l'entre- } \\
\text { preneur n'arrive pas à concrétiser } \\
\text { ses aspirations et attentes person- } \\
\text { nelles. Ce cas décrit la situation } \\
\text { dans laquelle l'échec de l'entre- } \\
\text { preneur a pour origine l'échec } \\
\text { personnel (exemple: sacrifice de } \\
\text { la vie personnelle pour assurer la } \\
\text { réussite professionnelle). }\end{array}$ & \\
\hline 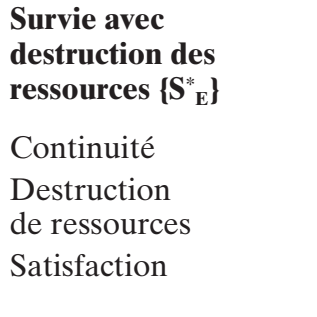 & $\begin{array}{l}\text { Malgré la faible performance éco- } \\
\text { nomique, qui s'explique en partie } \\
\text { par une mauvaise allocation des } \\
\text { ressources, l'entrepreneur retire } \\
\text { une satisfaction personnelle du } \\
\text { fait qu'en assurant la survie de } \\
\text { son entreprise «non rentable», } \\
\text { il concrétise des objectifs qui lui } \\
\text { sont propres. }\end{array}$ & \\
\hline
\end{tabular}




\section{La catégorie de scénarios $\mathrm{S}_{\mathrm{E}}^{*}$ ou «survie avec destruction de ressources»。} Cet espace d'échec recouvre les entrepreneurs qui ont choisi l'activité entrepreneuriale comme une source de réalisation de soi, d'épanouissement personnel (Maslow, 1943). Malgré la défaillance économique de son affaire, l'entreprise survit et l'entrepreneur retire une satisfaction personnelle du fait qu'il a atteint des objectifs qui lui permettent de se réaliser sur le plan personnel (reconnaissance par les autres, estime de soi, etc.).

En plus de ces différentes catégories d' «échec marginal» appartenant exclusivement aux espaces $\mathrm{S}_{\mathrm{D}}^{*} \mathrm{~S}_{\mathrm{I}}$ ou $\mathrm{S}_{\mathrm{E}}{ }_{\mathrm{E}}$, l'intersection de deux parmi les trois dimensions d'échec explicités précédemment permet de mettre en évidence les trois scénarios typologiques d'«échec partiel» (tableau 4): $\mathrm{S}_{\mathrm{EI}}, \mathrm{S}_{\mathrm{DE}}$ et $\mathrm{S}_{\mathrm{DI}}$.

\subsection{Les cas d'échec partiel}

La catégorie de scénarios $\mathbf{S}_{\mathrm{EI}}$ ou «survie marginale». Dans cette catégorie d'échec, on retrouve les petites entreprises émergentes qui parviennent à survivre et à franchir le cap des trois premières années. Cependant, elles n'arrivent ni à réaliser des rentes suffisantes ni à combler leur fondateur qui se trouve insatisfait car il ne réussit pas à réaliser ses ambitions. Bien qu'insatisfait sur la plan personnel et que son affaire soit déficitaire, l'entrepreneur persiste dans l'activité entrepreneuriale. Ce cas d'échec correspond généralement aux entreprises qui sont en état de cessation de paiement et qui échappent toujours à la faillite grâce à l'effort déployé et grâce aux ressources financières externes mobilisées par l'entrepreneur. Ce dernier est prêt à risquer ses propres biens pour maintenir en vie son affaire. Alors qu'il est insatisfait sur le plan personnel, le créateur ne prend pas la décision de mettre fin à son entreprise. La poursuite de l'activité est jugée par lui indispensable vu les engagements financiers et les efforts déployés. Bien qu'il n'en retire ni satisfaction personnelle, ni revenus suffisants, l'entrepreneur continue à lutter pour assurer la survie de son entreprise.

La catégorie de scénarios $S_{\mathrm{DE}}$ ou «sortie avec destruction de ressources». L'entrepreneur arrive à concrétiser ses aspirations et ses attentes personnelles. Cependant, l'entreprise en émergence ne génère pas de rente suffisante pour assurer sa survie. Bien que cette dernière ait disparu, son créateur a atteint ses objectifs personnels. C'est le cas, par exemple, des individus qui utilisent des fonds essentiellement d'origine bancaire pour réaliser des projets d'entreprises qui n'ont aucune chance de réussir. Les fonds sont utilisés à des fins personnelles et non pour assurer la croissance et la pérennité de leur entreprise. Ce cas d'échec est qualifié par Bruyat (1993) d'«escroquerie délibérée». Dans certains cas, le fondateur exclut du champ de ses aspirations 
les objectifs économiques pour privilégier des objectifs non économiques 3 . On peut aussi trouver dans cette catégorie des individus qui ont choisi la création comme une activité provisoire dans l'attente d'un emploi plus intéressant pour eux et dont l'entreprise n'est pas rentable sur le plan économique. Dès qu'ils trouvent une opportunité d'emploi plus intéressante, ils décident de vendre et/ou clôturer leur entreprise. Malgré la défaillance économique et la discontinuité de leur affaire, ils gagnent une activité provisoire et une expérience. Ce cas d'échec peut être qualifié de sortie positive sur le plan psychologique bien qu'il s'accompagne d'une destruction de ressources et d'une discontinuité entrepreneuriale.

La catégorie de scénarios $S_{\mathrm{DI}}$ ou «sortie avec déception de l'entrepreneur ». La nouvelle entreprise arrive à produire des richesses suffisantes pour assurer sa survie sous le contrôle de son fondateur. Cependant, la nouvelle entreprise disparaît. Cette mort n'est pas liée à une faillite lors d'une liquidation (mort par liquidation), mais c'est plutôt une mort sociologique. La nouvelle entreprise peut, par exemple, perdre son identité sociologique à la suite d'une situation obligeant l'entrepreneur à accepter une fusionabsorption (mort par fusion-absorption). En effet, les fusions-absorptions sont considérées par certains auteurs comme des morts d'organisation (Singh et Lumsden, 1990) dans la mesure où l'entreprise cesse d'exister sous son identité initiale. La mort sociologique peut aussi survenir lors d'un transfert de propriété à la suite d'une cession à une autre personne des parts sociales du fondateur, qui s'y trouve contraint par des variables personnelles, familiales et environnementales. L'entreprise n'est plus reconnue sous le nom de son fondateur mais sous celui du nouveau propriétaire. Dans les deux cas, le fondateur qui cherchait, à travers la création de son entreprise, à être son propre patron est déçu du fait qu'il n'arrive pas à satisfaire son besoin d'indépendance.

3. Cette manœuvre, qui est assimilable à une escroquerie, est utilisée par certains individus. Pour se protéger, ils ont souvent recours, dans certains pays, à des montages juridiques leur permettant de sauvegarder leurs biens personnels et familiaux en cas de faillite (prévisible, voire planifiée) de l'entreprise. 
TABLEAU 4

\section{Les cas d'échec partiel}

\begin{tabular}{|c|c|c|}
\hline Typologies d'échec & Définitions & Configurations \\
\hline $\begin{array}{l}\text { Survie marginale }\left\{\mathbf{S}_{\mathbf{E I}}\right\} \\
\text { Continuité } \\
\text { Destruction } \\
\text { de ressources } \\
\text { Insatisfaction }\end{array}$ & $\begin{array}{l}\text { Ce cas d'échec décrit la situation } \\
\text { au sein de laquelle la nouvelle } \\
\text { entreprise arrive à maintenir sa } \\
\text { survie mais le créateur est déçu } \\
\text { par la situation d'entrepreneur. } \\
\text { Cependant, malgré cette absence } \\
\text { de satisfaction personnelle, le } \\
\text { fondateur lutte pour maintenir } \\
\text { son affaire en vie. La décision de } \\
\text { cesser l'activité est jugée difficile } \\
\text { et la poursuite de l'activité est } \\
\text { jugée indispensable. }\end{array}$ & \\
\hline
\end{tabular}

Sortie avec destruction Malgré la défaillance éconode ressources $\left\{\mathbf{S}_{\mathrm{DE}}\right\}$ mique provoquant la discon-

Discontinuité

Destruction

de ressources

Satisfaction

\section{Sortie avec déception} de l'entrepreneur $\left\{S_{\mathrm{DI}}\right\}$

Discontinuité

Création de ressources Insatisfaction tinuité entrepreneuriale, le fondateur sort gagnant. Les fonds sont utilisés pour des fins personnelles et non pour assurer la croissance et la pérennité de son affaire. Cette catégorie d'échec correspond généralement au cas d'escroquerie.

L'entreprise nouvellement créée arrive à générer une rente suffisante pour assurer sa survie sous le contrôle de son fondateur. La discontinuité entrepreneuriale est liée à une transformation à l'occasion d'une fusion-absorption ou d'une cession de l'entreprise à
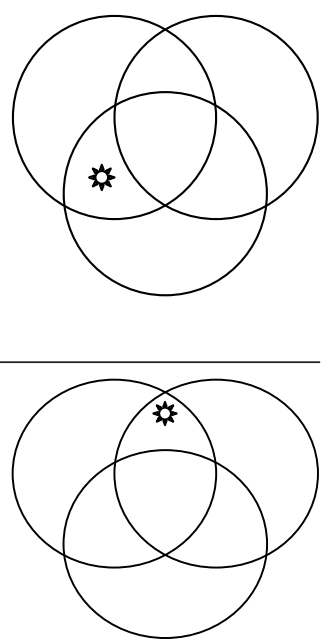
une tierce personne. L'entrepreneur qui cherchait, à travers la création de son entreprise, à être indépendant est insatisfait étant donné qu'il a perdu son autonomie à la suite de cette transformation. 


\section{Une illustration des catégories ou scénarios d'échec}

\subsection{Méthodologie de recherche}

\subsubsection{La méthode d'investigation choisie: les études de cas «collectives»}

Notre intention n'est pas de tester la véracité de la typologie proposée, mais de mieux percevoir l'échec entrepreneurial des petites entreprises émergentes en étudiant ses différentes configurations possibles. Pour parvenir à cet objectif, nous avons opté pour une démarche qualitative s'appuyant sur l'étude de cas dite «collective». Il s'agit d'un dispositif de recherche dans lequel plusieurs cas sont à étudier (David, 2003; Stake, 1995) simultanément. Comme le précise Stake (1995), l'étude de cas «collective» relève d'une approche «instrumentale» destinée à mieux circonscrire un phénomène à partir de cas multiples dans une optique plutôt exploratoire (David, 2003) que confirmatoire. Selon Hlady Rispal (2002, p. 78), cette approche «instrumentale» de l'étude des cas «est appropriée dans les situations où le chercheur veut illustrer des phénomènes préalablement définis dans un modèle théorique».

Cependant, l'ambition de cet article est non seulement d'illustrer les catégories ou scénarios d'échec en s'appuyant sur une approche «instrumentale », mais aussi d'essayer de garantir une analyse plus rigoureuse. Une double interrogation des cas par les théories et des théories par les cas est donc nécessaire (David, 2003). En s’inscrivant dans ce cadre méthodologique et en se référant à l'étude de Poroli (1999) portant sur la multidimensionnalité de la mort des organisations, les réflexions théoriques explicitées précédemment vont servir à étudier les cas. En retour, l'analyse de ces cas informe sur de nouvelles dimensions de l'échec entrepreneurial et nécessite un réexamen de la théorie. Un dépassement des cadres de références «classiques » et une ouverture de nouvelles perspectives théoriques deviennent alors possibles grâce à cet aller-retour entre l'observation empirique et l'analyse théorique.

\subsubsection{Constitution de l'échantillon des cas}

Afin d'illustrer les différentes configurations que peut revêtir l'échec d'une petite entreprise émergente, nous avons opté pour la constitution d'un échantillon sélectionné selon le critère de la variété. Par opposition au principe de la saturation théorique, qui est une caractéristique fondamentale de la théorie enracinée, la construction du terrain d'étude relève davantage d'une approche phénoménologique qui procède à un échantillonnage raisonné en termes de variété (Hlady Rispal, 2002, p. 87). Pour respecter ce principe 
d'échantillonnage, nous avons délibérément choisi des entrepreneurs ayant vécu des expériences d'échec variées. Les 10 cas que nous avons retenus nous semblent constituer un terrain d'étude convenable pour illustrer les scénarios d'échec (figure 2). Deux normes nous ont conduits à retenir ces entreprises :

- Eisenhardt (1989) recommande, afin d'être dans les normes d'une étude exploratoire, de retenir de 4 à 10 cas.

- Les entretiens préliminaires que nous avons menés auprès des témoins privilégiés (Quivy et Van Campenhoudt, 1995), c'est-à-dire des personnes ayant des connaissances spécifiques du phénomène étudié (accompagnateurs, experts comptables, dirigeants d'entreprises...), ont permis de retenir les cas les plus illustratifs de l'échec entrepreneurial dans le cas des petites entreprises émergentes.

\subsubsection{Le recueil des données}

Pour le recueil des données, nous avons privilégié, dans un premier temps, l'utilisation des entrevues non directives auprès des entrepreneur-fondateurs. Nous avons proposé au départ une seule et unique question: «Pouvez-vous exposer votre parcours, en commençant par le moment où vous avez envisagé de créer votre propre entreprise? », l'entrepreneur étant juste invité à parler de son aventure entrepreneuriale. La partie la plus difficile du recueil des données est celle portant sur les expériences d'échec vécues. Pour faire émerger les idées non exprimées par les entrepreneurs enquêtés à propos de leurs échecs, nous avons mené des entretiens semi-directifs. Pour répondre aussi au besoin d'opérationnalisation du concept «échec entrepreneurial», sur la base de la littérature qui nous a servi de «guide» pour aller sur le terrain, nous avons construit une grille d'analyse qui s'articule autour de deux dimensions non observables : la défaillance (ou la non-performance) économique et l'insatisfaction (ou la déception) personnelle de l'entrepreneur. Pour la dimension de la discontinuité, trois formes peuvent être distinguées: la discontinuité juridique (l'entreprise est juridiquement morte), la discontinuité économique (bien qu'immatriculée au registre de commerce, l'entreprise est économiquement inactive) et la discontinuité sociologique liée au remplacement de la personne du fondateur par une autre entité physique ou morale.

\subsection{Analyse des cas et synthèse des apports du terrain}

Nous avons analysé et synthétisé 10 cas de notre échantillon (voir annexe). Cette phase nous a aidés à répartir les entreprises étudiées dans les différents zones d'échec que la partie théorique de cet article a permis d'établir (tableau 5 et figure 2). 


\section{TABLEAU 5}

Composition de l'échantillon des cas selon les trois dimensions de l'échec

$\begin{array}{ccc}\begin{array}{c}\text { Discontinuité } \\ \text { entrepreneuriale }\end{array} & \begin{array}{c}\text { Défaillance } \\ \text { économique }\end{array} & \begin{array}{c}\text { Insatisfaction } \\ \text { de l'entrepreneur }\end{array}\end{array}$

Destruction

de ressources

\begin{tabular}{lllll}
\hline Cas A.K & $\mathbf{D}+$ & $\mathbf{O}$ & $\mathbf{I}+$ & $\mathbf{S}_{\mathrm{DI}}$ \\
Cas F.D & $\mathbf{O}$ & $\mathbf{E}+$ & $\mathbf{O}$ & $\mathbf{S}_{\mathbf{E}}^{*}$ \\
Cas F.K & $\mathbf{O}$ & $\mathbf{E}+$ & $\mathrm{I}+$ & $\mathrm{S}_{\mathrm{EI}}$ \\
Cas M.C & $\mathrm{O}$ & $\mathrm{O}$ & $\mathrm{I}+$ & $\mathrm{S}_{\mathrm{I}}^{*}$ \\
Cas M.N & $\mathrm{D}+$ & $\mathrm{E}+$ & $\mathrm{I}+$ & $\mathrm{S}_{\mathrm{DIE}}$ \\
Cas N.G & $\mathrm{D}+$ & $\mathrm{E}+$ & $\mathrm{I}+$ & $\mathrm{S}_{\mathrm{DIE}}$ \\
Cas R.K & $\mathrm{D}+$ & $\mathrm{E}+$ & $\mathrm{O}$ & $\mathrm{S}_{\mathrm{DE}}$ \\
Cas W.B & $\mathrm{D}+$ & $\mathrm{E}+$ & $\mathrm{O}$ & $\mathrm{S}_{\mathrm{DE}}$ \\
Cas W.K & $\mathrm{D}+$ & $\mathrm{O}$ & $\mathrm{I}+$ & $\mathrm{S}_{\mathrm{DI}}$ \\
Cas Z.K & $\mathrm{D}+$ & $\mathrm{O}$ & $\mathrm{O}$ & $\mathrm{S}_{\mathrm{D}}^{*}$ \\
\hline
\end{tabular}

Le signe (+) indique que la variable (concept) associée à la dimension correspondante de l'échec entrepreneurial est activée dans le discours des entrepreneurs enquêtés.

FIGURE 2

Répartition des cas de l'échantillon dans les différents scénarios d'échec

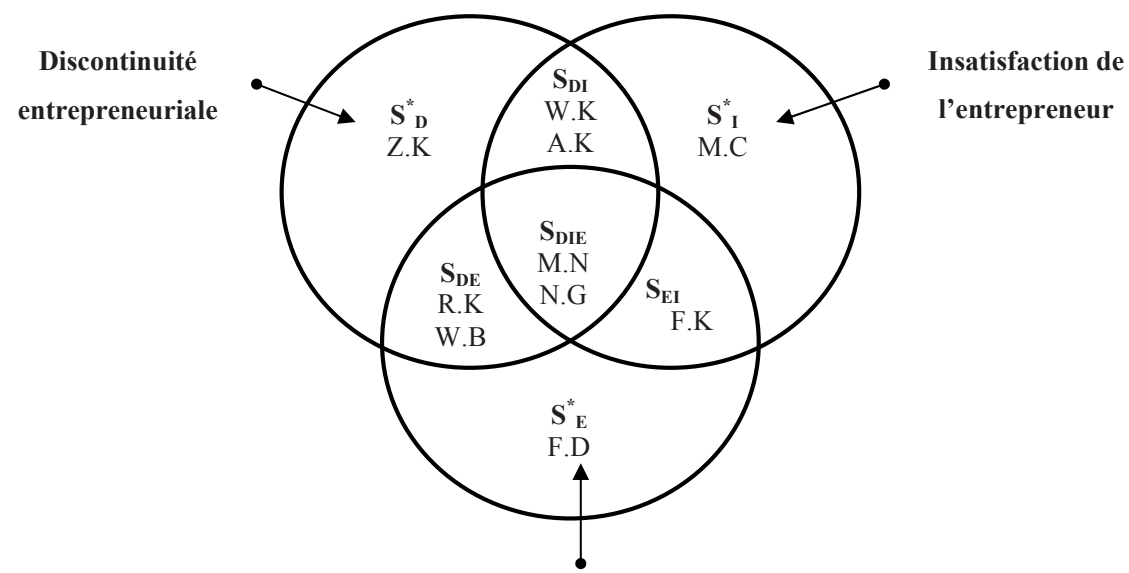

Défaillance économique

Destruction de ressources 
L'analyse des cas montre que l'échec entrepreneurial peut revêtir d'autres configurations que celle de la faillite. En effet, la forme la plus usuelle et extrême de l'échec est celle de la mort par liquidation lors d'une faillite (les cas M.N et N.G) qui correspond à la conjonction des trois dimensions: discontinuité, destruction des ressources et insatisfaction (figure 2). Les autres cas attestent la présence d'autres formes d'échec qui demeurent toutefois rarement abordées par la littérature. Par ailleurs, l'investigation des cas montre qu'il y a deux dimensions prépondérantes d'échec: économique et psychologique. C'est la conjonction de ces deux dimensions qui va conditionner le sort de la jeune petite entreprise: continuité versus discontinuité. Dans certaines circonstances, c'est l'entrepreneur qui va décider de poursuivre ou d'arrêter son aventure entrepreneuriale, dans d'autres circonstances, il est contraint d'arrêter cette aventure:

- pour les trois cas Z.K, R.K et W.B, la décision de la discontinuité est un choix délibéré plutôt qu'obligé;

- pour les autres cas de discontinuité (M.N, N.G, W.K et A.K), les entrepreneurs sont soumis à des obstacles économiques et/ou psychologiques qui les contraignent d'abandonner.

Cette approche décisionnelle de la discontinuité a été retenue par DeCastro et Szyliowicz (2004) pour distinguer entre «sortie volontaire » et «sortie involontaire». La première configuration regroupe l'ensemble des entrepreneurs qui sont soumis à des contraintes qui les forcent à mettre fin à leur aventure entrepreneuriale. La deuxième configuration regroupe les fondateurs qui conçoivent délibérément des «stratégies entrepreneuriales de sortie» (DeTienne et Cardon, 2006). Le créateur d'une entreprise peut en effet concevoir a priori une stratégie de sortie positive pour faciliter le passage à d'autres activités qu'il juge satisfaisantes pour lui, alors que son entreprise génère des bénéfices. Selon la perspective de la théorie de l'EPO, ce cas est interprété comme un échec. Cependant, selon la théorie de la «brèche aspirations-réalisations», il ne s'agit pas d'un échec puisqu'il se trouve en dehors de la zone $\left(\mathrm{S}_{\mathrm{I}}\right)$ d'insatisfaction. Le cas Z.K illustre cette forme de discontinuité (scénario $S_{D}{ }_{D}$ ) (figure 2). Pendant que son entreprise génère des bénéfices, Z.K a envisagé d'abandonner son affaire pour saisir l'opportunité d'un poste d'enseignant dans une école de commerce. Du fait qu'il a pu collecter des fonds suffisants pour financer la poursuite de ses études doctorales, Z.K exprime sa totale satisfaction.

La discontinuité de l'activité entrepreneuriale n'est donc pas toujours synonyme d'échec (Cope, Cave et Eccles, 2004; Everett et Watson, 1998; Shepherd, Douglas et Shanley, 2000; Shepherd et Zacharakis, 2001). Certains entrepreneurs décident de se retirer du marché pour des causes autres que 
l'insolvabilité. La discontinuité peut être parfois le résultat d'un succès et non la conséquence d'une mauvaise performance (Shepherd, Douglas et Shanley, 2000). Le cas A.K illustrant le scénario $S_{\mathrm{DI}}$ (figure 2) corrobore cette proposition. La réussite économique d'A.K attire dans le secteur des concurrents agressifs. L'un de ceux-ci s'arrangea, en utilisant son réseau relationnel, pour obtenir aux dépens d'A.K des quotas d'approvisionnement. Le concurrent était aussi motivé par l'appropriation du procédé de recyclage développé par A.K. Selon l'approche de l'échec par discontinuité entrepreneuriale, le cas A.K est vu comme un échec. Toutefois, selon l'approche fondée sur les ressources, ce cas est une réussite.

La discontinuité entrepreneuriale peut être encore due à des raisons autres que financières. Par exemple, des conflits interpersonnels avec les partenaires, en apparence mineurs, peuvent créer de sérieux problèmes conduisant finalement à une discontinuité (Singh, Corner et Pavlovich, 2007). Le cas W.K illustre cette forme de discontinuité (scénario $\mathrm{S}_{\mathrm{DI}}$ ) (figure 2). Malgré la réussite économique de son affaire (évolution positive du chiffre d'affaires et des bénéfices, récupération rapide de plus de $35 \%$ de l'investissement initial), W.K se trouve en conflit avec son partenaire. Déçu par le comportement de ce dernier, regrettant d'avoir perdu son autonomie de gestion, W.K décide de se retirer de l'affaire. Selon la théorie de ressources, ce cas est une réussite. Cependant, selon l'approche de l'échec par la théorie de la «brèche aspirations-réalisations », le cas W.K est un échec dans la mesure où l'entrepreneur n'a pas atteint ses objectifs de pouvoir et d'indépendance.

Notre approche de la notion de discontinuité conduit à distinguer «mortalité organisationnelle» et «discontinuité entrepreneuriale». La conception de discontinuité entrepreneuriale est plus large que celle de la mortalité organisationnelle puisqu'elle l'englobe. Shepherd, Douglas et Shanley (2000) avancent que la mort organisationnelle est spécifiquement liée à une baisse inattendue des recettes et/ou à une augmentation imprévisible des dépenses d'une ampleur telle que l'entreprise devient insolvable et incapable de s'endetter ou d'attirer de nouveaux capitaux: elle ne pourra plus continuer à opérer. Dans cette perspective, la mortalité apparaît comme le résultat d'un accident imprévu touchant la solvabilité de la nouvelle entreprise provoquant l'interruption de ses activités (Shepherd, 2003). La mortalité organisationnelle est donc un cas particulier de discontinuité entrepreneuriale. L'entrepreneur décide de mettre fin à son aventure entrepreneuriale uniquement pour des causes d'insolvabilité (Shepherd, Douglas et Shanley, 2000).

Le cas N.G illustre cette configuration d'échec (scénario $S_{\text {DIE }}$ ) (figure 2). Pour des raisons d'insolvabilité, N.G s'est trouvé dans un état de cessation de paiement. Confronté à une défaillance financière, il n'a plus rien à donner 
comme garantie bancaire, il a été acculé à la faillite. Selon la perspective de la théorie de l'EPO, l'échec est synonyme de mortalité. Cependant, la survie ou la «non-mortalité » de l'entreprise ne doit pas être systémiquement interprétée comme succès. Sur ce point, Honjo (2000) précise que l'échec ne comprend pas uniquement les entreprises qui ont été légalement déclarées en faillite, mais aussi les entreprises considérées comme inactives du point de vue économique malgré leur existence juridique. Selon l'approche de l'échec par la mortalité, les deux cas F.K et F.D illustrant respectivement les scénarios $\mathrm{S}_{\mathrm{EI}}$ et $\mathrm{S}_{\mathrm{E}}^{*}$ sont interprétés comme des réussites, dans la mesure où ces deux entreprises arrivent à survivre. Cependant, selon l'approche fondée sur les ressources, ces deux cas sont considérés comme des échecs.

Par ailleurs, en s'appuyant sur une approche de l'échec par la mortalité, on exclut de l'espace des échecs les cas qualifiés par Bruyat (1993) d'" escroquerie délibérée ». Le cas R.K illustrant le scénario $S_{\mathrm{DE}}$ est vu sous l'angle de la théorie de l'EPO comme un cas de succès car l'entreprise existe aujourd'hui par son immatriculation au registre de commerce. Cependant, selon la théorie des ressources, le cas R.K illustre un échec en termes de destruction de ressources. Les ressources de l'entreprise sont utilisées essentiellement pour des fins personnelles et non pour assurer la pérennité et la croissance de l'activité entrepreneuriale. R.K décide volontairement de «paralyser» son entreprise, ce qui la conduit à une «mort clinique».

Comme le souligne Valéau (2006), certains entrepreneurs passent par des moments de doute, de stress, de déception, d'hésitation entre la continuité et la discontinuité. Parmi eux, il y en a qui ont une motivation, une détermination et une volonté qui leur permettent de maintenir leur entreprise en vie malgré sa défaillance économique (cas F.K) ou malgré l'insuffisance de revenus qu'elle leur procure comparativement à ce que pourrait leur offrir le marché d'emploi s'ils étaient salariés (cas F.D) ou encore malgré un rythme ou style de vie qui ne leur convient pas du tout (cas M.C). D'autres, par contre, envisagent la cessation de l'activité entrepreneuriale pour des raisons d'insolvabilité (les cas M.N et W.K), pour éviter des pertes économiques (cas W.B) ou pour mettre fin à des pressions psychologiques qui les épuisent mentalement (les cas W.K et A.K). En plus de l'aspect économique, l'échec entrepreneurial est aussi associé à des états psychologiques de démotivation, de découragement, de manque de persévérance et d'endurance. Les origines de cette catégorie d'échec peuvent être mêlées d'insatisfaction personnelle $\mathrm{du}$ fondateur à la suite de la non-concrétisation de ses attentes initiales et/ou de l'entrée de sa jeune petite entreprise dans la spirale de défaillance économique qui le démotive. 


\section{Conclusion}

Le recours à une approche intégrative mobilisant la théorie d'écologie des populations des organisations, l'approche fondée sur les ressources et la théorie de la «brèche aspirations-réalisations » permettent de proposer une analyse conceptuelle et typologique de l'échec entrepreneurial. À l'aide de la conjonction des trois dimensions d'échec (discontinuité entrepreneuriale, destruction de ressources et déception personnelle du fondateur), cette recherche a réussi à distinguer les différentes catégories ou scénarios d'échec et a pu les illustrer grâce à des études de cas «collectives». Cette investigation, fondée d'abord sur un cadre théorique intégrateur puis sur l'analyse de 10 cas de petites entreprises émergentes, a montré que l'échec de ce type d'entreprise peut revêtir d'autres configurations que celle de la faillite. La mort par liquidation lors d'une faillite légale est la forme la plus extrême d'échec. Les résultats de cette étude permettent aussi de dépasser la dichotomie classique succès/échec. Entre ces deux extrêmes qui sont plutôt théoriques que réels, il y a des configurations intermédiaires qui demeurent rarement abordées par la littérature et que le présent article a essayé d'explorer, de caractériser et de catégoriser.

Les données qualitatives analysées nous ont permis de mieux cerner les «symptômes» d'un échec entrepreneurial et de délimiter les contours du phénomène à trois dimensions: la dimension économique, qui s'exprime en termes de destruction de ressources, la dimension psychologique, qui se mesure à travers l'insatisfaction personnelle de l'entrepreneur, et la dimension sociologique, liée à la discontinuité du projet entrepreneurial sous le leadership du fondateur. L'analyse des cas montre que le fondateur peut décider la continuité ou la discontinuité, mais il peut également être contraint de mettre fin à son entreprise. Dans certaines circonstances, l'entrepreneur est un acteur qui conduit volontairement son entreprise à la disparition. Il conclut alors soit à une sortie positive pour saisir d'autres opportunités plus intéressantes pendant que son entreprise génère des bénéfices ou avant d'atteindre le seuil critique des pertes, soit à une mort clinique à la suite d'une escroquerie planifiée. Dans d'autres circonstances, c'est le contexte entrepreneurial qui dicte à l'entrepreneur la trajectoire à suivre. Dans cette perspective, les entrepreneurs sont confrontés à des contraintes économiques (état d'insolvabilité irrémédiable) et psychologiques (état de stress insupportable) qui les obligent à abandonner.

Au plan scientifique, cet article contribue à une meilleure compréhension du concept d'échec entrepreneurial en proposant une typologie qui met en évidence les catégories ou les scénarios d'échec possibles. La typologie proposée apporte un éclairage permettant de comprendre et d'analyser les 
situations d'échec. Elle apporte par ailleurs des enseignements supplémentaires sur des situations qui sont parfois paradoxales. Bien que centrée sur les petites entreprises émergentes, la typologie élaborée par cette étude couvre les différentes configurations possibles de l'échec entrepreneurial. Par rapport à la typologie usuelle qui oppose les entreprises en bonne santé aux entreprises défaillantes, la typologie avancée dans cet article intègre une dimension psychologique relevant davantage de la déception du fondateur à la suite de la non-concrétisation de ses aspirations et attentes initiales. Par rapport aux travaux existants (Bruyat, 1993; Paturel, 2007) qui insistent surtout sur la réussite, cet article met l'accent sur l'échec entrepreneurial. Aux dimensions déjà abordées par les auteurs (réussite du projet d'entreprise, réussite du projet de vie de l'entrepreneur, efficacité, efficience et effectivité) que cet article aborde sous forme de destruction de ressources et d'insatisfaction de l'entrepreneur, cet article enrichit la typologie de l'échec par la dimension de discontinuité entrepreneuriale.

L'intérêt managérial de cette recherche est susceptible de se révéler auprès de plusieurs acteurs. La typologie proposée peut aider les consultants, les accompagnateurs, les banquiers... en leur permettant de faire une analyse des différentes configurations d'échec. Les manifestations économiques et psychologiques et les symptômes spécifiques qui traduisent chaque situation d'échec pourraient aider à remonter aux causes. Comprendre ces échecs et leurs causes pourrait donc aider les acteurs professionnels et institutionnels à développer des stratégies préactives d'évitement ou réactives de redressement, ou encore proactives créant l'événement de sortie positive. Ces stratégies sont propres à chaque scénario mis en évidence par la typologie développée par cet article. Sur le terrain, bien qu'il reste du chemin à parcourir, la typologie avancée peut être ultérieurement déclinée en tant qu'outil de management anticipatif pour aider les entrepreneurs à définir leurs choix stratégiques. Concrètement, ces choix stratégiques peuvent tenir compte des rapports ou ratios entre les motivations (économiques et non économiques) de l'entrepreneur, les ressources (financières, technologiques, humaines, etc.) et les contraintes contextuelles à affronter (vivacité de la concurrence, absence d'un marché réel et solvable, etc.). La présence de la nouvelle entreprise dans l'une des situations d'échec signale un déséquilibre entre ces grandeurs, ce qui nécessite une intervention d'acteurs externes notamment les accompagnateurs et les consultants pour aider l'entrepreneur à sortir de la spirale de l'échec entrepreneurial.

Cependant, cet article connaît des limites qui, d'ailleurs, peuvent être des points de départ pour des recherches ultérieures. D'abord, la typologie proposée hérite naturellement des limites des travaux de recherche sur lesquels elle se fonde. Il faut noter, à ce niveau, que les recherches citées 
ne s'intéressent pas aux entreprises nouvellement créées de petite taille, mais aux nouvelles entreprises en général. Certaines situations d'échec peuvent donc ne pas être exclusivement associées aux petites entreprises émergentes. Ensuite, le caractère exploratoire de la présente recherche fait que la typologie proposée ne peut pas dans l'état actuel de la recherche faire l'objet d'une généralisation. Par ailleurs, la typologie présentée est statique et néglige l'aspect dynamique du phénomène de l'échec entrepreneurial. Une piste de recherche pourrait être l'exploration d'études de cas supplémentaires dans le but d'avoir une analyse plus approfondie conduisant à mieux asseoir la typologie proposée dans cet article. Une autre piste de recherche serait d'identifier les trajectoires types d'échec. Ces deux pistes de recherche contribueraient à une approche dynamique qui pourrait mieux répondre à la question du «comment de l'échec entrepreneurial».

\section{ANNEXE Synthèse des 10 cas de l'échantillon}

\section{Le cas A.K illustrant le scénario $S_{D I}$ de "sortie avec déception de l'entrepreneur"}

Attiré par les avantages fiscaux et financiers accordés par les pouvoirs publics pour encourager les jeunes promoteurs à créer leur propre entreprise, A.K décide de se lancer dans l'aventure entrepreneuriale. L'absence d'un emploi stable suffisamment rémunéré est aussi l'une des motivations qui le poussent à choisir l'entrepreneuriat. C'est dans l'industrie du recyclage des déchets en plastique qu'A.K souhaite investir. Pour acquérir l'expertise nécessaire, A.K suit une formation professionnelle. Au début de 2006, A.K crée son entreprise qu'il dénomme SRDS. Mais, dès le début,A.K se trouve confronté à plusieurs problèmes. L'obstacle majeur qui menace la survie de son projet entrepreneurial est la limitation de quotas d'approvisionnement en déchets plastiques imposée par les autorités de tutelle. Malgré cette contrainte, le chiffre d'affaires et les bénéfices de SRDS progressent. A.K élargit alors son domaine d'activité pour inclure, en plus du recyclage, les activités d'injection, d'extrusion et de soufflage des déchets en plastique. Toutefois, A.K reste soumis à deux pressions qui, selon lui, sont à l'origine d'un stress permanent et insupportable:1) sa famille «harcèle» pour qu'il abandonne son activité et intègre la fonction publique. Cela est dû à deux caractéristiques culturelles des pays de A.K (la famille intervient fortement dans les projets de vie de ses membres; être fonctionnaire est plus sûr et plus valorisant qu'être un petit entrepreneur);2) son concurrent, en utilisant son réseau relationnel, peut 
obtenir aux dépens de A.K des quotas d'approvisionnement et procédé de production. C'est sous ces pressions épuisantes qu'A.K se trouve finalement dans l'obligation de céder son projet d'entreprise à son rival. La situation d'A.K est alors: insatisfaction personnelle et abandon de l'activité entrepreneuriale, malgré une réussite économique.

\section{Le cas F.D illustrant le scénario $S_{E}^{*}$ de survie avec une performance économique médiocre}

Pour F.D, l'idée de créer sa propre entreprise est le fruit des conseils de son ami et de son frère qui disposent respectivement d'une expérience dans le secteur de l'imprimerie et d'une expérience dans le domaine de la papeterie. Sa propre formation de base n'ayant aucun lien avec l'activité d'emballage et d'infographie de l'entreprise qu'il veut créer, F.D commence par effectuer des stages techniques dans ce domaine. Pour financer le démarrage de son entreprise, il investit des fonds provenant de son épargne personnelle. Optant pour une stratégie de croissance prudente, F.D refuse l'idée de financer le développement de son projet d'entreprise par des emprunts bancaires. Malgré la forte demande du marché, F.D se contente de quelques clients provenant de son entourage immédiat. Malgré l'insuffisance de revenus comparés à ce que pourrait lui offrir le marché de travail s'il était salarié, F.D exprime sa totale satisfaction. Il avance que son objectif n'est pas de faire fortune mais de gagner ce qu'il faut pour une vie décente. La situation de F.D est alors: continuité entrepreneuriale et satisfaction personnelle du fondateur, en dépit de la faible performance économique de son entreprise.

\section{Le cas F.K illustrant le scénario $S_{\mathrm{E}}$ de survie marginale}

Au bout de trois ans d'activité salariale pour le compte d'un laboratoire de photographie, F.K juge qu'il dispose d'une expérience suffisante pour créer sa propre entreprise dans le secteur de l'impression numérique. Sa décision de quitter son activité salariale est aussi motivée par une réelle volonté d'autonomie et d'indépendance. Pour financer son projet et acquérir un matériel à la pointe de la technologie, F.K s'endette auprès de son entourage familial. Mais très vite, plusieurs problèmes se posent: mauvaise gestion, saturation du marché régional, guerre des prix exercée par des concurrents agressifs, clients insolvables, fonds de roulement insuffisant, tension de trésorerie...F.K se trouve alors dans l'impossibilité de rembourser ses dettes. Il s'ensuit des conflits entre F.K et son entourage familial à cause du non-remboursement de la dette. Pour essayer de sortir de cette situation, F.K est obligé de brader une partie de son matériel high-tech. Quatre ans après le démarrage de son affaire, F.K se trouve dans une situation financière difficile avec un marché 
réduit à un petit quartier de la ville, avec moins de matériel performant, un chiffre d'affaires lui permettant juste de couvrir les charges et de maintenir son entreprise affaiblie en vie. Il est insatisfait de sa situation personnelle et familiale, ses attentes n'ont pas été réalisées. Mais la pression sociale (le regard des autres, son environnement culturel) le pousse à ne pas mettre fin à son aventure entrepreneuriale. La situation de F.K est alors: continuité entrepreneuriale, destruction de ressources (échec économique) et insatisfaction quant à la réalisation des attentes initiales.

\section{Le cas M.C illustrant le scénario $S^{*}$ de survie avec déception de l'entrepreneur}

Après avoir occupé pendant plusieurs années un poste de responsable technique dans une entreprise de construction métallique, M.C crée sa propre entreprise en métallurgie afin de profiter des compétences acquises tout au long de son travail salarial. Encouragé par les bénéfices qu'il réalise assez rapidement sur le marché local, M.C décide de se lancer assez vite dans l'export. À cause d'une mauvaise préparation de cette phase et de sa méconnaissance du marché international, M.C se trouve soumis à de très fortes pressions: stress, déplacement fréquent, vie familiale décousue, échec scolaire de ses enfants, etc. Son rêve qu'une activité entrepreneuriale allait lui permettre une vie qui échappe aux pressions exercées par ses supérieurs hiérarchiques est brisé par une vie de stress et d'échecs familiaux. Son entreprise qui est pérenne et se développe est une réussite économique, mais il est complètement insatisfait de sa situation en tant que chef d'entreprise. La situation de M.C est alors: continuité entrepreneuriale, création de ressources, mais insatisfaction de l'entrepreneur.

\section{Le cas M.N illustrant le scénario $S_{\text {DIE }}$ d'échec total}

Dès l'obtention de son mastère, M.N se lance dans la réalisation du rêve qui l'a accompagné pendant sa vie d'étudiant: créer sa propre entreprise. Il s'agit d'une pâtisserie industrielle visant l'ensemble du marché national grâce à une logistique performante s'appuyant sur une chaîne de froid dans un pays chaud. Confronté à une lourdeur bureaucratique et à des problèmes financiers et techniques, le démarrage de l'entreprise n'a lieu que quatre ans plus tard. Mais pendant cette période, plusieurs paramètres ont énormément évolué mettant en péril la faisabilité du projet: forte augmentation des prix de la matière plastique de conditionnement, du coût de matériel de production, de l'intensité concurrentielle du secteur de la pâtisserie entraînée par une floraison d'hôtels dans les zones touristiques du pays. L'investissement finalement engagé atteint le double de ce qui était initialement prévu, d'où un surendettement de M.N. En plus, M.N se trouve fortement dépendant 
d'un nombre limité de clients qui vont se révéler insolvables. Les lourdes charges salariales qui ont également connu une hausse notable aggravent la dégradation de la situation financière de l'entreprise. Face à une situation de cessation de paiement qu'il juge insurmontable, M.N se trouve envahi par des sentiments d'impuissance, de désespoir, de déception et de démotivation. Épuisé mentalement (déception et démotivation) et physiquement, M.N tombe malade. Il décide alors de mettre fin à son entreprise deux ans après son démarrage. La situation de M.N est alors: discontinuité de son entreprise, destruction des ressources et insatisfaction.

\section{Le cas W.B illustrant le scénario $\mathrm{S}_{\mathrm{DE}}$ de sortie avec destruction de ressources}

Après des études d'ingénieur en art et communication, W.B décide de créer sa propre entreprise, qu'il dénomme V.S, dans le secteur de la communication publicitaire. Pour se différencier de la concurrence, W.B se spécialise dans la communication visuelle en $3 \mathrm{D}$ et plus précisément dans les visites virtuelles des immeubles, une offre destinée aux promoteurs immobiliers. Au démarrage, W.B est confronté à des obstacles d'ordre commercial (difficultés de joindre les clients et de les convaincre de l'utilité de son offre) qui s'expliquent en partie par la culture de la clientèle régionale. L'entreprise V.S se trouve alors dans une situation d'insolvabilité. Mais les contacts que W.B réussit à tisser lui permettent de rencontrer un promoteur hôtelier d'envergure internationale. Celui-ci finit par confier à W.B un poste qui non seulement valorise son savoir-faire dans la communication $3 \mathrm{D}$, mais lui ouvre aussi des perspectives d'épanouissement car il lui laisse une grande liberté pour exercer sa créativité. W.B décide alors de mettre fin à son activité entrepreneuriale pour reprendre un poste qu'il juge tout à fait satisfaisant. La situation de W.B est alors: discontinuité entrepreneuriale et destruction des ressources, mais satisfaction personnelle.

\section{Le cas Z.K illustrant le scénario $\mathbf{S}_{\mathrm{D}}$ de sortie positive}

Z.K vient d'obtenir son mastère d'informatique. Son rêve est de continuer ses études doctorales dans une université étrangère très réputée. Mais, faute de moyens financiers, il renonce provisoirement à cette idée. Avec le soutien d'un ami qui lui facilite l'obtention d'un crédit fournisseur pour l'acquisition de micro-ordinateurs, Z.K crée une entreprise. Il s'agit d'un club informatique pour enfants. Son affaire a si bien marché qu'au bout de deux ans il réussit à la vendre à un groupe d'écoles d'informatique pour enfants, en réalisant une forte plus-value. Cela lui permet de s'inscrire à l'université dont il a rêvé. Il y soutient son doctorat et il y est même embauché en tant que professeur. 
Z.K considère qu'il a tiré toute satisfaction de son entreprise. La situation de Z.K est alors: discontinuité entrepreneuriale malgré une création de ressources, mais satisfaction de l'entrepreneur.

\section{Le cas N.G illustrant le scénario $S_{\text {DIE }}$ d'échec total}

Après avoir obtenu son diplôme de maîtrise en commerce international, N.G se trouve au chômage. Il décide alors de faire appel au soutien financier pour créer une petite entreprise dans la maintenance et la vente de matériel informatique ainsi que la conception des CD-ROM (c'est l'entreprise I.P). En plus de son désir de cesser de chômer, sa motivation s'appuyait sur son désir d'indépendance. Mais assez vite et pour de multiples raisons (mauvaise gestion des ressources financières, charges d'exploitation trop élevées, saturation du marché local...), l'entreprise I.P connaît des difficultés financières. Pour échapper à une faillite, N.G a recours à des découverts bancaires et sollicite des aides financières auprès de son entourage personnel. Malgré les efforts déployés, l'entreprise I.P devient «une machine à fabriquer des pertes». Face à cette situation, N.G devient constamment habité par un sentiment d'impuissance à l'égard de l'avenir de sa jeune entreprise. Confronté à un état de cessation de paiement, N.G est obligé de liquider son entreprise et de trouver assez rapidement un emploi stable de salarié. La situation de N.G est alors: discontinuité entrepreneuriale, destruction de ressources et insatisfaction de l'entrepreneur.

\section{Le cas R.K illustrant le scénario $S_{D E}$ de sortie avec destruction des ressources}

Propriétaire d'une petite imprimerie qu'il vient de créer, R.K décide de créer une entreprise de distribution de la production de sa propre entreprise. Deux facteurs sont à l'origine de cette démarche: R.K accorde une importance primordiale aux signes extérieurs de richesse pour gagner l'admiration de son milieu social (être le patron de deux entreprises) et veut échapper à l'imposition fiscale (la création de la nouvelle entreprise lui offre le bénéfice des déductions fiscales offertes par la réglementation en vigueur). Il s'endette alors auprès de sa banque pour financer son nouveau projet entrepreneurial. Prisonnier de sa logique du paraître (signes extérieurs de richesse), il utilise une partie de ces fonds pour des dépenses personnelles. Malgré la situation financière alarmante de sa nouvelle entreprise qui finit par cesser toute activité mais reste immatriculée au registre de commerce, R.K exprime sa totale satisfaction du fait qu'il mène un train de vie luxueux. Sur le plan juridique, la nouvelle entreprise continue à exister, mais, sur le plan économique elle est dans un état de «mort clinique». La situation de R.K est alors: discontinuité entrepreneuriale, destruction de ressources et satisfaction de l'entrepreneur. 


\section{Le cas W.K illustrant le scénario $S_{D I}$ de sortie avec déception de l'entrepreneur}

Âgé de 28 ans, W.K juge que toutes les conditions sont réunies pour créer sa propre entreprise et réaliser ainsi son désir d'indépendance (être son propre patron): une formation et une expérience relativement solides (assistance technique, réparation du matériel informatique, formation spécialisée en informatique); des ressources financières épargnées pendant sa période d'activité salariée dans une société informatique; une opportunité perçue lors de son travail salarié, compatible avec ses compétences et aptitudes. Il s'associe alors avec un technicien en informatique et démarre la nouvelle entreprise. Ce démarrage est fulgurant puisqu'il permet de récupérer assez rapidement plus de $35 \%$ de la mise initiale. Mais aussitôt un conflit portant sur la répartition des bénéfices l'oppose à son associé. Ce conflit ne trouve aucune solution, vu l'incompatibilité des caractères des deux protagonistes. Habitué à travailler dans la sécurité et la stabilité de son emploi initial, W.K juge qu'il ne peut plus supporter le stress et décide, six mois après la date de démarrage et malgré la réussite économique de son projet, de céder son entreprise à son associé. Ce dernier, qui au départ ne détenait qu'une faible part du capital, se trouve aujourd'hui le nouveau propriétaire. L'entreprise a désormais changé de statut juridique. La situation de W.K est alors: discontinuité entrepreneuriale, création de ressources et insatisfaction de l'entrepreneur.

\section{Bibliographie}

Abdesselam, R., J. Bonnet et N. LE PAPE (2004), «An explanation of the life span of new French firms », Small Business Economics, vol. 23, n 3, p. 237-254.

AMit, R. et E. Muller (1994), «"Push" and "Pull” entrepreneurship», Frontiers of Entrepreneurship Research, Babson College.

ArRÈGLE, J.L. (2006), «Analyse "Resource Based" et identification des actifs stratégiques », Revue française de gestion, vol. 32, n 160, p. 241-259.

Aspelund, A., T. Berg-Utby et R. SkJEVdal (2005), «Initial resources influence on new venture survival: a longitudinal study of new technology-based firms », Technovation, vol. 25, n ${ }^{\circ} 11$, p. 1337-1347.

BACQ, S., O. GIACOMIN et F. JANSSEN (2009), «L'échec et la seconde chance», dans F. JANSSEN (dir.), Entreprendre, une introduction à l'entrepreneuriat, Bruxelles, De Boeck, p. 255-264.

BAldwin, J., L. BiAn, R. Dupuy et G. Gellaty (2000), Taux d'échec des nouvelles entreprises canadiennes: nouvelles perspectives sur les entrées et les sorties, Ministre de l'Industrie, Statistique Canada.

BARNEY, J. (1991), «Firm resources and sustained competitive advantage », Journal of Management, vol. 17, $\mathrm{n}^{\circ}$ 1, p. 99-120. 
BRUTON, G.D. et Y. RUBANIK (2002), «Resources of the firm, Russian high-technology start-ups, and firm growth », Journal of Business Venturing, vol. 17, n ${ }^{\circ} 6$, p. 553-576.

BRUYAT, C. (1993), Création d'entreprise: contributions épistémologiques et modélisation, Thèse de doctorat en sciences de gestion, Université Pierre-MendèsFrance, Grenoble II.

BRUYAT, C. (2001), «Créer ou ne pas créer? Une modélisation du processus d'engagement dans un projet de création d'entreprise », Revue de l'Entrepreneuriat, vol.1, n ${ }^{\circ} 1$, p. $25-42$.

CAnnon, M. et A.C. Edmondson (2001), «Confronting failure: antecedents and consequences of shared beliefs about failure in organizational work groups », Journal of Organizational Behaviour, vol. 22, nº 2, p. 161-177.

CAPIEZ, A. et E.M. HernANDEZ (1998), «Vers un modèle d'émergence de la petite entreprise », Revue internationale PME, vol. 11, n 4, p. 11-43.

Chrisman, J.J., A. BAuerschmidT et C.W. Hofer (1999), «The determinants of new venture performance: an extended model», Entrepreneurship Theory and Practice, vol. 22, n ${ }^{\circ}$ 1, p. 5-29.

COOPER, A.C. et W.A. ARTZ (1995), «Determinants of satisfaction for entrepreneurs», Journal of Business Venturing, vol. 10, n ${ }^{\circ}$ 6, p. 439-457.

COOPER, A.C., F.J. GiMENO-CACSON et C.Y. Woo (1994), «Initial human and financial capital as predictors of new venture performance», Journal of Business Venturing, vol. 9, n 5 , p. 371-395.

COOPER, A.C., J. GASCON et C. WOO (1991), «A resource-based prediction of new venture survival and growth», Academy of Management Proceedings, p. 113-119.

COPE, J., F. CAVE et S. ECCLES (2004), «Attitudes of venture capital investors towards entrepreneurs with previous business failure», Venture Capital, vol. 6, n ${ }^{\text {os }} 2-3$, p. $147-172$.

Crutzen, N. et D. VAn CAillie (2008a), «Vers une typologie des micros et petites entreprises en difficulté: une étude exploratoire», XVII ${ }^{\mathrm{e}}$ Conférence internationale de Management stratégique, Nice.

Crutzen, N. et D. VAN CAillie (2008b), «Le processus de défaillance de l'entreprise : intégration et relecture de la littérature », IX ${ }^{\mathrm{e}}$ Conférence du CIFEPME, Louvain-la-Neuve.

CRutzen, N. et D. VAn CAillie (2009), «Vers une taxonomie des profils d'entrée dans un processus de défaillance: un focus sur les micro- et petites entreprises en difficulté », Revue internationale PME, vol. 22, n 1, p. 101-128.

DAhlqVist, J., P. DAVIDSSON et J. WiKLUND (2000), «Initial conditions as predictors of new venture performance: a replication and extension of the Cooper et al. study», Enterprise \& Innovation Management Studies, vol. 1, n 1, p. 1-17.

DANJOU, I. (2002), «L'entrepreneuriat un champ fertile à la recherche de son unité», Revue française de gestion, vol. 28, nº 138, p. 109-386. 
DAVID, A. (2003), «Étude de cas et généralisation scientifique en sciences de gestion», Revue sciences de gestion, $\mathrm{n}^{\circ}$ 39, p. 139-166.

DeCAStro, J. et D. SzYLiowicz (2004), «Entrepreneurial failure? The factors that predict voluntary vs. involuntary exit», Frontiers of Entrepreneurship Research, Babson College.

DETIENNE, D. et M.C. CARDON (2006), «Entrepreneurial exit strategies : the impact of human capital», Frontiers of Entrepreneurship Research, Babson College.

EISENHARDT, K.M. (1989), «Building theories from case study research», The Academy of Management Review, vol. 14, n 4, p. 532-550.

EVERETT, J. et J. WATSON (1998), «Small business failure and external risk factors », Small Business Economic, vol. 11, no 4, p. 371-390.

Fotopoulos, G. et H. Louri (2000), «Location and survival of new entry», Small Business Economic, vol. 4, no 4, p. 311-321.

FRITSCH, M., U. BRIXY et O. FALCK (2006), «The effect of industry, region, and time on new business survival - a multi-dimensional analysis », Review of Industrial Organization, vol. 28, n 3, p. 285-306.

GRAY, R.K., H. Foster et M.HOWARD (2006), «Motivation of Moroccans to be entrepreneurs », Journal of Developmental Entrepreneurship, vol. 11, n 4, p. 297-318.

Guilhot, B. (2000), «Défaillances d'entreprise: soixante-dix ans d'analyses théoriques et empiriques », Revue française de gestion, $\mathrm{n}^{\circ}$ 130, septembre-octobre, p. 52-67.

HANNAN, M.T. et J. FREEMAN (1977), «The population ecology of organisations », American Journal of Sociology, n 82, vol. 5, p. 929-964.

HEADD, B. (2003), «Redefining business success : distinguishing between closure and failure», Small Business Economics, vol. 21, no 1, p. 51-61.

Hernandez, E.M. et L. MARCo (2002), «L'entrepreneuriat et les théories de la firme », Revue française de gestion, vol. 28, n 138, p. 127-144.

HLADY RisPAL, M. (2002), La méthode des cas: application à la recherche en gestion, Bruxelles, De Boeck, 256 p.

HONJO, Y. (2000), «Business failure of new firms : an empirical analysis using a multiplicative hazards model», International Journal of Industrial Organization, vol. $18, \mathrm{n}^{\circ} 4$, p. 557-574.

JenNings, P.L. et G. BEAVER (1995), «The managerial dimension of small business failure », Journal of Strategic Change, vol. 4, n 4, p. 185-200.

KHELIL, N. (2006), «Une approche de l'irrationalité par les risques. Application à la décision entrepreneuriale de création d'entreprises ", Actes de la Ve Conférence de l'Association internationale et interdisciplinaire de la Décision, Tozeur, Tunisie.

LASCH, F., F. LE ROY et S. YAMI (2005), «Les déterminants de la survie et de la croissance des start-ups TIC», Revue française de gestion, vol. 31, n 155, p. 37-56.

LELOGEAIS, L. (2004), «Le rôle des variables qualitatives dans la détection précoce du risque de défaillance », XIII Conférence de l'AIMS, Normandie. 
LEVINTHAL, D.A. (1991), «Random walks and organizational mortality », Administrative Science Quarterly, vol. 36, nº 3, p. 397-420.

LEVy-TAdjine, T. et R. PATUREL (2006), «Essai de modélisation trilogique du phénomène entrepreneurial», dans C. Fourcade, G. Paché et R. Pérez (dir.), La stratégie dans tous ses états, Éditions EMS - Management et Société, p. 311-321.

Littunen, H., E. Storhammar et T. NenOnen (1998), «The survival of firms over the critical first 3 years and the local environment», Entrepreneurship \& Regional Development, vol. 10, n 3, p. 189-202.

LUSSIER, R.N. (1995), «A nonfinancial business success versus failure prediction model for young firms », Journal of Small Business Management, vol. 33, n 1, p. 8-20.

LUSSIER, R.N. (1996), «A start-up business success versus failure prediction model for the retail industry», The Mid-Atlantic of Business, vol. 32, n 2, p. 79-92.

LUSSIER, R.N. et S. PFEIFER (2001), «A crossnational prediction model for business success », Journal of Small Business Management, vol. 39, n 3, p. 228-239.

MALÉCOT, J.F. (1991), «Analyse historique des défaillances d'entreprises: une revue de la littérature », Revue d'économie financière, vol. 19, p. 205-226.

Maslow, A.H. (1943), "A theory of human motivation», Psychological Review, vol. 50, p. 370-396.

MCGRATH, R.G. (1999), «Falling forward: real options reasoning and entrepreneurial failure », Academy of Management Review, vol. 24, n 1, p. 13-30.

MELlAHI, K. et A. WiLKINSON (2004), «Organizational failure: a critique of recent research and a proposed integrative framework», International Journal of Management Reviews, vol. 5-6, n 1, p. 21-41.

Michalos, A.C. (1986), «Job satisfaction, marital satisfaction, and the quality of life: a review and preview», dans F.M. Andrews (dir.), Research on the Quality of Life, Ann Arbor, University of Michigan Institute for Social Research.

Mignon, S. (2001), Stratégie de pérennité d'entreprise, Paris, Vuibert.

Moreau, R. (2007), «La réussite entrepreneuriale: proposition pour un modèle explicatif général», Gestion 2000, vol. 24, n 3, p. 45-62.

Morgan, G. (2006), Images of Organizations, édition mise à jour, Thousand Oaks, Cal., Sage Publications.

MurPHY, G.B. et S.K. CALlAWAY (2004), «Doing well and happy about it ? Explaining variance in entrepreneurs' stated satisfaction with performance », New England Journal of Entrepreneurship, vol. 7, no 2, p. 15-27.

Ouedraogo, A. (1999), Les déterminants de la création de PME dans un pays d'Afrique sub-saharienne: une analyse quadridimensionnelle du phénomène entrepreneurial au Burkina-Faso, Thèse de doctorat en sciences de gestion, Université de Caen Basse-Normandie.

PAPADAKI, E. et B. CHAMI (2002), Les facteurs déterminants de la croissance des micro-entreprises au Canada, Direction générale de la politique de la petite entreprise Industrie Canada.

PATUREL, R. (1997), Pratique du management stratégique, Grenoble, Presses universitaires de Grenoble. 
PATUREL, R. (2007), «Démarche stratégique et performance de la PME», dans L.J. Filion (dir.), Management des PME: de la création à la croissance, Montréal, Éditions du Renouveau pédagogique, p. 429-443.

POROLI, C. (1999), «La multidimensionnalité de la mort des organisations», Cahier $n^{\circ} 274$, Centre de recherche DMSP Dauphine Marketing Stratégie prospective.

QUIVY, R. et L.VAN CAMPENHOUDT (1995), Manuel de recherche en sciences sociales, $2^{\mathrm{e}}$ édition, Paris, Dunod.

RAY, D.M. et D.V. TRUPIN (1989), «A crossnational comparison of entrepreneur's perceptions of success », Entrepreneurship \& Regional Development, vol. 1, $\mathrm{n}^{\circ} 1, \mathrm{p} .113-127$.

REID, C. (1999), «Complex actions and simple questions : how new entrepreneurs stay in business?», Small Business Economics, vol. 13, n 4, p. 303-315.

SAMMUT, S. (1995), Contribution à la compréhension du processus de démarrage en petite entreprise, Thèse de doctorat en sciences de gestion, Montpellier.

SHANE, S., E.A. LoCKE et C.J. COLLINS (2003), «Entrepreneurial motivation», Human Resource Management Review, vol. 13, n 2, p. 257-279.

SHELTON, L.M. (2005), «Scale barriers and growth opportunity: a resource-based model of new venture expansion», Journal of Enterprising Culture, vol. 13, $\mathrm{n}^{\circ} 4$, p. 333-357.

SHEPHERD, D.A. (2003), «Learning from business failure: propositions about the grief recovery process for the self-employed», Academy of Management Review, vol. $28, \mathrm{n}^{\circ} 2$, p. 318-329.

SHEPHERD, D.A. et A. ZACHARAKIS (2001), «The venture capitalist-entrepreneur relationship : control, trust and confidence in co-operative behavior», Venture Capital, vol. 3, n 2, p. 129-149.

Shepherd, D.A., E.J. Douglas et M. Shanley (2000), «New venture survival: ignorance, external shocks, and risk reduction strategies », Journal of Business Venturing, vol. 15, $\mathrm{n}^{\text {os }}$ 5-6, p. 393-410.

SINGH, J.V. et C.J. LUMSDEN (1990), «Theory and research in organizational ecology », Annual Review of Sociology, vol. 16, n 1, p. 161-195.

SinGH, S., P. CORNER et K. PAVLOVICH (2007), «Coping with entrepreneurial failure», Journal of Management \& Organization, vol. 13, n 4, p. 331-344.

SMIDA, A. (1992), Prospective des métamorphoses du système technique et management des ruptures technologiques, Thèse de doctorat d'État en sciences de gestion, Université de Caen Basse-Normandie.

SMIDA, A. (1995), «Ingrédients de prospective et leviers de stratégie», dans A. Noël, P. Véry et M. Wissler (dir.), Perspectives en management stratégique, Tome III, Economica, p. 455-483.

SMIDA, A. (2003), «Décisions dans un univers de contraintes: approches préactives, réactives et proactives», dans B. Cadet, C. Grenier et A. Smida (dir.), Les décisions sous contraintes, Caen, Presses universitaires de Caen, p. 363-377. 
SmidA, A. (2006a), «Les moments de décision stratégique. Un essai de conceptualisation et de modélisation », $\mathrm{XV}^{\mathrm{e}}$ Conférence internationale de Management stratégique, Annecy/Genève.

SMIDA, A. (2006b), «L'irrationalité au service de la stratégie», Ve Colloque de l'Association internationale et interdisciplinaire de la Décision, Tozeur, Tunisie.

SMIDA, A. (2007), «SMOCS, un modèle de management anticipatif stratégique pour piloter l'organisation dans des environnements complexes », VI ${ }^{\mathrm{e}}$ Colloque international de l'Association tunisienne des sciences de gestion, Hammamet, Tunisie.

StAke, R.E. (1995), The Art of Case Study Research, Thousand Oaks, Cal., Sage Publications.

SteArns, T.M., N.M. CARTER, P.D. Reynolds et M.L. Williams (1995), «New firm survival: industry, strategy and location », Journal of Business Venturing, vol. $10, \mathrm{n}^{\circ} 1$, p. $23-43$.

STINCHCOMBE, A.L. (1965), «Social structure and organizations», dans J.G. March (dir.), Handbook of Organizations, Chicago, Rand McNally, p. 142-193.

SUTTON, R.I. (1987), «The process of organizational death: disbanding and reconnecting », Administrative Science Quarterly, vol. 32, no 4, p. 542-569.

TABLEAU DE L'ÉCONOMIE FRANÇAISE (2006), Institut national de la statistique et des études économiques.

THORNHILL, S. et R. AMIT (2003), «Comprendre l'échec: mortalité organisationnelle et approche fondée sur les ressources », Document de recherche $n^{\circ}$ 202, Division de l'analyse microéconomique, Statistique Canada.

VALÉAU, P. (2006), «L'accompagnement des entrepreneurs durant les périodes de doute», Revue de l'Entrepreneuriat, vol. 5, nº 1, p. 31-57.

VAN PRAAG, C.M. (2003), «Business survival and success of young small business owner», Small Business Economics, vol. 21, n 1, p. 1-17.

Venkataraman, S., A.H. VAn de Ven, J. Buckeye et R. Hudson (1990), «Starting up in a turbulent environment: a process model of failure among firms with high customer dependence», Journal of Business Venturing, vol. 5, n 5, p. 277-295.

WATKINS, D. (1976), «Entry into independent entrepreneurship: toward a model of the business initiation process », Working Paper Series, Manchester Business School and Center for Business Research.

WERnERFELT, B. (1984), «A resource-based view of the firm », Strategic Management Journal, vol. 5, no 2 , p. 171-180.

WiKLund, J. et D.A. SHEPHERD (2001), «Intentions and growth: the moderating role of resources and opportunities », Academy of Management Proceedings.

Zacharakis, AL., GD. MeYer et J. DeCASTRo (1999), «Differing perceptions of new venture failure: a matched exploratory study of venture capitalists and entrepreneurs », Journal of Small Business Management, vol. 37, n 3, p. 1-14. 